\title{
Modelling strong interactions and longitudinally polarized vector boson scattering
}

\author{
Adam Falkowski, ${ }^{a b}$ Stefan Pokorski ${ }^{b}$ and J.P. Roberts ${ }^{b}$ \\ ${ }^{a}$ CERN Theory Division, \\ CH-1211 Geneva 23, Switzerland \\ ${ }^{b}$ Institute of Theoretical Physics, Warsaw University, \\ Hoża 69, 00-681 Warsaw, Poland \\ E-mail: adam.falkowski@cern.ch, Stefan.Pokorski@fuw.edu.pl, \\ roberts@fuw.edu.pl
}

ABSTRACT: We study scattering of the electroweak gauge bosons in 5D warped models. Within two different models we determine the precise manner in which the Higgs boson and the vector resonances ensure the unitarity of longitudinal vector boson scattering. We identify three separate scales that determine the dynamics of the scattering process in all cases. For a quite general background geometry of $5 \mathrm{D}$, these scales can be linked to a simple functional of the warp factor. The models smoothly interpolate between a 'composite' Higgs limit and a Higgsless limit. By holographic arguments, these models provide an effective description of vector boson scattering in $4 \mathrm{D}$ models with a strongly coupled electroweak breaking sector.

Keywords: Technicolor and Composite Models, Higgs Physics, Large Extra Dimensions. 


\section{Contents}

1. Introduction 1

2. Gauge boson scattering in the Standard Model 3

3. Parameterising strongly coupled electroweak sectors 国

4. Holographic composite Higgs 6

4.1 Mass eigenstates 0

4.2 Scales 9

4.3 Goldstone bosons 11

4.4 Couplings of resonances 11

5. Holographic pseudo-Goldstone Higgs 14

5.1 Mass eigenstates 14

5.2 Scales 16

5.3 Goldstone bosons 17

5.4 Couplings to resonances 18

6. Gauge boson scattering amplitudes 19

7. Conclusions 21

A. Warped trigonometry 23

B. $\mathrm{SU}(3)_{c} \times \mathrm{SU}(2)_{L} \times \mathrm{SU}(2)_{R} \times \mathrm{U}(1)_{X}:$ profiles and couplings

G. $\mathrm{SU}(3)_{c} \times \mathrm{SO}(5) \times \mathrm{U}(1)_{X}$ : profiles and couplings 27

\section{Introduction}

The mechanism of electroweak symmetry breaking is still unknown. Within the SM, electroweak symmetry is broken by the condensation of a weakly coupled elementary scalar field, the Higgs field. This simple mechanism is consistent with electroweak precision measurements if the mass of the Higgs boson is within the range $100-200 \mathrm{GeV}$. However any such fundamental scalar that is much lighter than the SM cut-off is unnatural as there is no symmetry protecting its mass.

There are two primary approaches to solving this hierarchy problem of the SM. The first, supersymmetry, provides a rationale for elementary scalars and protects the Higgs boson masses from large quantum corrections. The simplest implementation - the MSSM - 
ensures perturbative physics up to the Planck scale and provides several interesting predictions at the $\mathrm{TeV}$ scale. Unfortunately it is not free from some residual tuning of parameters once experimental constraints are imposed.

A radically different idea is that a new, strongly interacting, sector provides a $\mathrm{TeV}$ scale cut-off to the SM. One can envisage Higgsless electroweak symmetry breaking that is generated in a manner similar to the chiral symmetry breaking in QCD. The longitudinal components of the $W$ and $Z$ bosons are provided by three Goldstone bosons of the strongly interacting sector. However breaking electroweak symmetry with a strongly interacting sector does not necessarily lead to a Higgsless theory. It is also possible to construct models in which the full Higgs doublet emerges as a composite particle. An interesting subset of such models are those in which the composite Higgs doublet arises as a pseudo-Goldstone boson of some spontaneously broken approximate global symmetry of the strongly interacting theory.

Strongly interacting theories are notoriously difficult to handle in four dimensions. It has been suggested [1], however, that they may have a 'holographic dual' description in terms of a 5D gauge theory in a warped background [2]. Modelling strong interactions by $5 \mathrm{D}$ theories has become a useful tool, allowing for quantitative studies of both QCD [3] and electroweak symmetry breaking [ : 5 ,

It is well known [6], and has been recently emphasised in [4, 8] that scattering of longitudinally polarized $W$ and $Z$ bosons may be used as a probe of the dynamics that breaks electroweak symmetry. Therefore in this paper we use the calculation of the $W$ and $Z$ boson scattering amplitudes to analyse and compare different $5 \mathrm{D}$ descriptions of the electroweak symmetry breaking sector.

It is interesting to systematize the phenomenology of this holographic approach. In this paper we extract the common features that show up in in gauge boson scattering that are independent of such details of the model building as the symmetries of the strongly interacting sector or the warp factor describing the $5 \mathrm{D}$ geometry. The recurring feature is the appearance of three distinct physical scales:

- $v$ : the electroweak breaking scale that sets the mass of $\mathrm{W}$ and $\mathrm{Z}$.

- $f_{h}$ : the scale that sets the compositeness scale of the Higgs, referred to as the Higgs decay constant.

- $M_{\mathrm{KK}}$ : the resonance scale that sets the mass of the first resonance .

How these scales emerge from the $5 \mathrm{D}$ dual is a model dependent question. However, we show that the separation between these scales does not depend on the fine-grained details of the model. More precisely one can define a simple functional, which we call the volume factor, that depends on the size and the geometry of the 5th dimension. The volume factor fixes the ratio $f_{h} / M_{\mathrm{KK}}$ and, in the Higgsless case, also $v / M_{\mathrm{KK}}$. The same volume factor also fixes $\Lambda / M_{\mathrm{KK}}$ where $\Lambda$ is the strong coupling scale at which the $5 \mathrm{D}$ effective description breaks down.

We discuss in some detail how the aforementioned scales show up in the gauge boson scattering amplitudes. In the Standard Model the exchange of a Higgs boson cancels the 
divergent behaviour of the four point gauge boson vertex. This cancellation is not maintained in more complex models of electroweak symmetry breaking. As can be expected, the violation of unitarity is associated with the scale $f_{h}$ that controls departures of the Higgs couplings from the Standard Model, while the full restoration of unitarity is postponed until the resonance scale $M_{\mathrm{KK}}$. We present quantitative results for the scattering amplitudes in two different 5D models. One is the model or ref. [9] describing a composite Higgs emerging from a strong sector with the $\mathrm{SO}(4)$ custodial symmetry. The other is the model of ref. [5] describing a pseudo-Goldstone Higgs from breaking $\mathrm{SO}(5) \rightarrow \mathrm{SO}(4)$ by strong interactions.

We also consider the Higgsless limit of the 5D models. This is the limit where the Higgs boson decouples from the electroweak bosons and plays no role in restoring unitarity, even though it may remain in the physical spectrum. In this case the electroweak scale $v$ becomes intimately tied to the geometry of the 5th dimension and equals $f_{h}$. The Higgsless limit turns out to be particularly insensitive to the details of 5D modelling.

The paper is structured as follows. In section 2 we review the unitarisation of the gauge boson scattering amplitudes in the SM. We employ the equivalence theorem that allows us to calculate the scattering in terms of scattering of the Goldstone bosons eaten by $W$ and $Z$. This serves to highlight the role of the Higgs boson in the unitarisation and to fix our notation for the rest of the paper. In section 3 we discuss in general terms the manner in which strongly coupled electroweak sectors affect the longitudinal vector boson scattering. In section 1 we turn to modelling a strongly interacting electroweak breaking sector using a 5D holographic dual. We investigate the 5D model proposed in [9] with the Higgs sector localized on the IR brane and custodial symmetry in the bulk. We calculate the couplings of the Goldstone bosons to the physical Higgs and to the resonances, and employ useful approximations that reveal a simple pattern in these couplings. In section 5 , we repeat this program for a 5D model of the pseudo-Goldstone Higgs [5]. In section 6 we collect the results of our quantitative studies and use them to calculate the precise form of the WZ scattering amplitude. We present our conclusions in section 7. Three appendices contain more technical details of our computations.

\section{Gauge boson scattering in the Standard Model}

First we review the unitarisation of longitudinal gauge boson scattering in the SM. Here we use the equivalence theorem (ET) to calculate the scattering amplitudes via the Goldstone bosons 10. This serves to fix our notation, and to highlight the role of the Higgs boson in unitarising the amplitudes.

The Lagrangian for the Higgs doublet is:

$$
\mathcal{L}=\left|\partial_{\mu} H\right|^{2}-V\left(H^{\dagger} H\right)
$$

We parameterise the Higgs fields non-linearly:

$$
H=\frac{1}{\sqrt{2}}(v+h) U\left(\begin{array}{l}
0 \\
1
\end{array}\right),
$$


where $\quad U=e^{\left(\frac{i G_{a} \sigma_{a}}{v}\right)}=\cos \left(\frac{G}{v}\right)+i \frac{G_{a} \sigma_{a}}{G} \sin \left(\frac{G}{v}\right), \quad G^{2}=G_{a} G_{a}$

where $h$ is the physical Higgs boson, $v$ is the Higgs vev and $G_{a}$ are the three Goldstone bosons. Inserting this into our Lagrangian (2.1) we get:

$$
\mathcal{L}=\frac{1}{2}\left(\partial_{\mu} h\right)^{2}-V(h)+\frac{1}{2}\left(1+\frac{h}{v}\right)^{2}\left[\left(\partial_{\mu} G\right)^{2}+\frac{\sin ^{2}(G / v)}{(G / v)^{2}}\left(\left(\partial_{\mu} G_{a}\right)^{2}-\left(\partial_{\mu} G\right)^{2}\right)\right]
$$

From this we acquire canonically normalized kinetic terms and the interaction terms of the Goldstone bosons and the Higgs boson that we need to calculate the scattering. The relevant terms are:

$$
\begin{aligned}
\mathcal{L}_{G^{4}} & =\frac{1}{6 v^{2}}\left(\left(G_{a} \partial_{\mu} G_{a}\right)^{2}-\left(\partial_{\mu} G_{a}\right)^{2} G_{b} G_{b}\right) \\
\mathcal{L}_{G^{2} h} & =\frac{h}{v}\left(\partial_{\mu} G_{a}\right)^{2}
\end{aligned}
$$

To complete the picture we introduce the $\mathrm{SU}(2)_{L} \times \mathrm{U}(1)_{Y}$ gauge fields. The $W^{ \pm}$bosons acquire longitudinal polarizations by eating the Goldstone modes $G^{ \pm}=\left(G_{1} \mp i G_{2}\right) / \sqrt{2}$ while the $\mathrm{Z}$ boson eats $G_{3}$. Moreover, there appear three-point vertices involving gauge bosons:

$$
\begin{aligned}
\mathcal{L}= & -i\left(G^{-} \partial_{\mu} G^{+}-G^{+} \partial_{\mu} G^{-}\right)\left(g_{\gamma} A_{\mu}+g_{Z} Z_{\mu}\right)-i\left(G_{3} \partial_{\mu} G^{-}-G^{-} \partial_{\mu} G_{3}\right) g_{W} W_{\mu}^{+} \\
& -i\left(G_{3} \partial_{\mu} G^{+}-G^{+} \partial_{\mu} G_{3}\right) g_{W} W_{\mu}^{-}
\end{aligned}
$$

where $g_{\gamma}=e, g_{Z}=\left(g_{L}^{2}-g_{Y}^{2}\right) / 2 \sqrt{g_{L}^{2}+g_{Y}^{2}}, g_{W}=g_{L} / 2$ and $g_{L}, g_{Y}$ are the SM gauge couplings.

The Higgs boson plays a crucial role in unitarising scattering processes in which the initial and final state particles are $W$ or $Z$. Using the ET we can calculate the leading order contribution to the relevant scattering processes using the following amplitudes for Goldstone boson scattering. Here we take the process $W_{L} Z_{L} \rightarrow W_{L} Z_{L}$ as an example:

$$
\mathcal{M}_{G^{+} G^{3} \rightarrow G^{+} G^{3}}=\frac{t}{v^{2}}-\frac{t}{v^{2}} \frac{t}{t-m_{h}^{2}}-g_{W}^{2}\left(\frac{t-s}{u-m_{W}^{2}}+\frac{t-u}{s-m_{W}^{2}}\right)
$$

Via the ET, this amplitude corresponds to the amplitude for $W_{L}^{ \pm} Z_{L} \rightarrow W_{L}^{ \pm} Z_{L}$, up to terms $\mathcal{O}\left(m_{W} / E\right)$. The first term grows quadratically with energy. This leads to unitarity violation at high energies, unless it is cancelled by the term from Higgs boson exchange that follows. Therefore the presence of a sufficiently light Higgs boson restores unitarity in the theory. The last term from the $W$ boson exchange is irrelevant to the discussion of quadratic divergences as it contributes no growing term, but we have included it for later convenience.

\section{Parameterising strongly coupled electroweak sectors}

We now go on to examine the question of the unitarity of gauge boson scattering in theories with an extended electroweak sector. In the SM unitarisation of the gauge boson scattering 
amplitude relies on the cancellation between the quartic Goldstone vertex and the Higgs exchange diagrams. This requires a precise correlation between the quartic Goldstone selfcoupling and the Higgs-Goldstone coupling. In the following we will discuss 5D models of electroweak symmetry breaking and investigate how they affect this correlation.

Since 5D warped physics provides a holographic description of strongly coupled theories, we expect that deviations from the SM scattering amplitudes will depend directly upon the compositeness scale of the Higgs boson, which we denote as $f_{h}$. The composite structure should reveal itself in modifications of Higgs-Goldstone couplings by terms of order $1 / f_{h}^{2}$. We thus expect the couplings to have the form:

$$
\mathcal{L}_{G^{2} h}=g_{h} \frac{h}{v}\left(\partial_{\mu} G^{a}\right)^{2}
$$

where $g_{h}=1-\mathcal{O}\left(v^{2} / f_{h}^{2}\right)$. For $g_{h}<1$ the Higgs boson on its own cannot unitarise the scattering of the gauge bosons:

$$
\mathcal{M}_{G^{+} G^{3} \rightarrow G^{+} G^{3}} \approx \frac{t}{v^{2}}-g_{h}^{2} \frac{t}{v^{2}} \frac{t}{t-m_{h}^{2}}
$$

Above the scale of the Higgs mass we obtain $\mathcal{M}_{G^{+} G^{3} \rightarrow G^{+} G^{3}} \sim t / f_{h}^{2}$. The amplitude continues growing up to $M_{\mathrm{KK}}$ where some other physics (e.g. vector resonances) must restore unitarity. The Higgless case corresponds to $g_{h}=0$.

As recently discussed [7], this kind of behaviour is expected on purely low energy grounds if the Higgs doublet arises as a pseudo-Goldstone boson. Consider the case of SO(5) broken to $\mathrm{SO}(4)$ where we identify the remaining $\mathrm{SO}(4)$ with the approximate $\mathrm{SU}(2)_{L} \times$ $\mathrm{SU}(2)_{R}$ custodial symmetry of the SM. The four Goldstone bosons are identified with the Higgs doublet. The Lagrangian at lowest order is:

$$
(1 / 2) \partial_{\mu} U^{T} \partial_{\mu} U
$$

with $U$ parameterising the Goldstone bosons:

$$
U=f_{h} \exp \left(\frac{i \sqrt{2} H_{a} T_{a}}{f_{h}}\right)\left(\begin{array}{l}
\overrightarrow{0} \\
1
\end{array}\right)
$$

Here $T_{a}$ are the four broken generators of $\mathrm{SO}(5)$ and $H_{a}$ are the four Goldstone bosons of the broken symmetry that we identify with the Higgs fields. Finally, $f_{h}$ is the scale of the global symmetry breaking. The Higgs field gets a vev $\left\langle H_{4}\right\rangle=\tilde{v}$. The electroweak breaking scale is related to its vev by $v=f_{h} \sin \left(\tilde{v} / f_{h}\right)$.

To calculate scattering amplitudes we need to extract the three point couplings of two Goldstone bosons to the Higgs boson. Parameterising the Higgs field as in eq. (2.2) and expanding the lowest order Lagrangian we find the relevant Higgs-Goldstone couplings:

$$
\mathcal{L}_{G^{2} h}=\cos \left(\frac{\tilde{v}}{f_{h}}\right) \frac{h}{v}\left(\partial_{\mu} G_{a}\right)^{2}
$$

Thus $g_{h}=\cos \left(\tilde{v} / f_{h}\right)$. The four-point couplings remain unchanged from (2.4). Therefore, above the Higgs boson mass the WZ amplitude grows as $\sim\left(1-\cos ^{2}\left(\tilde{v} / f_{h}\right)\right) t / v^{2}=t / f_{h}^{2}$. 
This is the simplest setup in which the non-unitary behaviour is encountered, irrespectively whether the high-energy UV completion that restores unitarity is perturbative or strongly coupled.

In the following sections we shall study 5D warped models from the point of view of gauge boson scattering. We will find similar qualitative behaviour, even when the Higgs is not a pseudo-Goldstone boson. We will also study in detail how the vector resonances restore unitarity of the scattering amplitudes.

\section{Holographic composite Higgs}

We first consider a $5 \mathrm{D}$ theory with the gauge symmetry $\mathrm{SU}(3)_{c} \times \mathrm{SU}(2)_{L} \times \mathrm{SU}(2)_{R} \times$ $\mathrm{U}(1)_{X}$ and the Higgs field on the IR brane, which was proposed in ref. [9]. The limit where the brane Higgs vev goes to infinity corresponds to the Higgsless theory of ref. 11. The rationale behind extending the $\mathrm{SM} \mathrm{U}(1)_{Y}$ to $\mathrm{SU}(2)_{R} \times \mathrm{U}(1)_{X}$ is to avoid excessive contributions to the $\mathrm{T}$ parameter [9, 12].

The 5th dimension is warped with a gravitational background described by the line element:

$$
d s^{2}=a^{2}\left(x_{5}\right) \eta_{\mu \nu} d x^{\mu} d x^{\nu}-d x_{5}^{2}
$$

where $a\left(x_{5}\right)$ is a warp factor normalized such that $a(0)=1$. The 5 th dimension is bounded by two branes, an IR brane at $x_{5}=L$ and a UV brane at $x_{5}=0$. The choice $a\left(x_{5}\right)=1$ corresponds to flat space, whereas $a\left(x_{5}\right)=e^{-k x_{5}}$ corresponds to $\mathrm{AdS}_{5}$. We do not specify the warp factor in what follows other than to require that it generate a sufficient hierarchy between the UV and the IR brane: $a(L) \equiv a_{L} \ll 1$. Moreover, we will assume that the size $L$ of the extra dimension is large in the sense $L \frac{\partial_{5} a(L)}{a_{L}} \gg 1$. This is typically the case in backgrounds that solve the hierarchy problem such as $\mathrm{AdS}_{5}$. The significance of this last assumption will become clear in the following.

We allow the gauge bosons to propagate in the bulk, while the Higgs sector is confined to the IR brane. On the UV brane we explicitly break the gauge symmetry down to the SM gauge group.

This set-up can be interpreted as an effective description of a 4D theory with fundamental SM gauge bosons and a strongly coupled electroweak symmetry breaking sector with a global $\mathrm{SU}(3)_{c} \times \mathrm{SU}(2)_{L} \times \mathrm{SU}(2)_{R} \times \mathrm{U}(1)_{X}$ symmetry. The IR brane Higgs boson is interpreted as a composite of the strongly interacting sector.

The $5 \mathrm{D}$ action for the electroweak sector is:

$$
\begin{aligned}
S= & \int d^{4} x \int_{0}^{L} d x_{5} \sqrt{g}\left\{-\frac{1}{2} \operatorname{Tr}\left(L_{M N} L_{M N}\right)-\frac{1}{2} \operatorname{Tr}\left(R_{M N} R_{M N}\right)-\frac{1}{4} X_{M N} X_{M N}\right\} \\
& +\int d^{4} x d x_{5} \sqrt{g_{4}} \delta(L)\left(\frac{1}{4} \operatorname{Tr}\left|D_{\mu} \Phi\right|^{2}-V\left(\Phi^{\dagger} \Phi\right)\right)
\end{aligned}
$$

where $L_{M N}$ and $R_{M N}$ are the $\mathrm{SU}(2)_{L, R}$ gauge fields respectively, $X_{M N}$ are the $\mathrm{U}(1)_{X}$ gauge fields and $\Phi$ are the scalar fields that we identify with the Higgs. The dimensionful couplings of $\mathrm{SU}(3)_{c} \times \mathrm{SU}(2)_{L} \times \mathrm{SU}(2)_{R} \times \mathrm{U}(1)_{X}$ will be denoted as $g_{L} \sqrt{L}, g_{R} \sqrt{L}$ and $g_{X} \sqrt{L}$. 
The Higgs field acquires a vev $\langle\Phi\rangle=a_{L}^{-1} \tilde{v} I_{2 \times 2}$ that spontaneously breaks $\mathrm{SU}(2)_{L} \times$ $\mathrm{SU}(2)_{R} \rightarrow \mathrm{SU}(2)_{V}$ on the IR brane. We separate out the physical Higgs boson from the Goldstone bosons by parameterising the Higgs boson non-linearly:

$$
\begin{aligned}
\Phi & =a_{L}^{-1}(\tilde{v}+h) U \\
\text { with } \quad U & =\exp \left(\frac{i \tilde{G}_{a} \sigma_{a}}{\tilde{v}}\right)=\cos \left(\frac{\tilde{G}}{\tilde{v}}\right)+i \frac{\tilde{G}_{a} \sigma_{a}}{\tilde{G}} \sin \left(\frac{\tilde{G}}{\tilde{v}}\right)
\end{aligned}
$$

where $\tilde{G}^{2}=\tilde{G}_{a} \tilde{G}_{a}$ and $h$ is the physical Higgs boson. From the covariant derivative of the Higgs field we get the quadratic terms:

$$
\mathcal{L}=\frac{1}{2} \delta(L)\left[\partial_{\mu} \tilde{G}_{a}-\frac{\sqrt{L} \tilde{v}}{2}\left(g_{L} L_{\mu, a}-g_{R} R_{\mu, a}\right)\right]^{2}+\frac{1}{2} \delta(L)\left(\partial_{\mu} h\right)^{2}
$$

These terms provide a kinetic term for the Higgs boson and the Goldstone bosons as well as a brane mass term for the gauge bosons. They also introduce the mixing between the Goldstone bosons and the gauge bosons.

\subsection{Mass eigenstates}

The dynamics of the model can be neatly studied in the mass eigenstate formalism introduced in [13, 12]. We expand the 5D fields in the mass eigenstate basis:

$$
\begin{aligned}
L_{\mu}^{a}\left(x, x_{5}\right) & =A_{\mu, n}(x) f_{L, n}^{a}\left(x_{5}\right) & & L_{5}^{a}\left(x, x_{5}\right)=G_{n}(x) \bar{f}_{L, n}^{a}\left(x_{5}\right) \\
R_{\mu}^{a}\left(x, x_{5}\right) & =A_{\mu, n}(x) f_{R, n}^{a}\left(x_{5}\right) & & R_{5}^{a}\left(x, x_{5}\right)=G_{n}(x) \bar{f}_{R, n}^{a}\left(x_{5}\right) \\
X_{\mu}\left(x, x_{5}\right) & =A_{\mu, n}(x) f_{X, n}\left(x_{5}\right) & & X_{5}\left(x, x_{5}\right)=G_{n}(x) \bar{f}_{X, n}\left(x_{5}\right) \\
\tilde{G}_{n}(x) & =G_{n}(x) \tilde{f}_{n} & &
\end{aligned}
$$

where the index $n$ runs over all mass eigenstates in the theory. The profiles $f_{n}\left(x_{5}\right)$ will be chosen such that the gauge bosons are indeed mass eigenstates. The Goldstone profiles $\bar{f}_{n}\left(x_{5}\right)$ will be chosen such that $G_{n}$ becomes the Goldstone boson corresponding to the massive eigenstate $A_{\mu, n}$. In other words, the goal is to rewrite the quadratic part of the $5 \mathrm{D}$ action as a $4 \mathrm{D}$ action that is diagonal in $n$ :

$$
S_{5}=\int d^{4} x \sum_{n}\left\{-\frac{1}{4}\left(\partial_{\mu} A_{\nu, n}-\partial_{\nu} A_{\mu, n}\right)^{2}+\frac{1}{2}\left(\partial_{\mu} G_{n}-m_{n} A_{\mu, n}\right)^{2}\right\}+\text { interactions . }
$$

In this way, there is no tree-level mixing between the light modes and the heavy KK modes, even in the presence of electroweak symmetry breaking on the brane. This is different from the more common approach, where the KK expansion is performed in the absence of electroweak symmetry breaking, and the electroweak symmetry breaking leads to mixing of zero modes with KK modes.

We also retain the Goldstone degrees of freedom. The Goldstones, $G_{n}$, allow us to maintain explicit gauge invariance in the presence of the mass term for the vector $A_{\mu, n}$. Keeping Goldstones is convenient as, via the equivalence theorem, scattering of longitudinally polarized vector bosons $A_{\mu, n}$ is equivalent to scattering of $G_{n}$.

In order to end up with the diagonal action (4.6) the profiles $f_{n}\left(x_{5}\right)$ must satisfy: 
1. The equation of motion

$$
\left[\partial_{5}\left(a^{2} \partial_{5}\right)+m_{n}^{2}\right] f_{n}\left(x_{5}\right)=0
$$

2. The orthonormality condition

$$
\int_{0}^{L} f_{n}(y) f_{m}(y)=\delta_{n m}
$$

3. The $U V$ boundary conditions:

$$
\begin{aligned}
\partial_{5} f_{L, n}^{a}(0) & =0 & & a=1,2,3 \\
f_{R, n}^{i}(0) & =0 & & i=1,2 \\
s_{x} \partial_{5} f_{R, n}^{3}(0)+c_{x} \partial_{5} f_{X, n}(0) & =0 & & s_{x}=\frac{g_{X}}{\sqrt{g_{X}^{2}+g_{R}^{2}}} \\
-c_{x} f_{R, n}^{3}(0)+s_{x} f_{X, n}(0) & =0 & & c_{x}=\frac{g_{R}}{\sqrt{g_{X}^{2}+g_{R}^{2}}}
\end{aligned}
$$

which break $\mathrm{SU}(2)_{R} \times \mathrm{U}(1)_{X}$ down to $\mathrm{U}(1)_{Y} \cdot^{1}$

4. The IR boundary conditions

$$
\begin{aligned}
\partial_{5} f_{X, n}(L) & =0 \\
g_{R} \partial_{5} f_{L, n}^{a}(L)+g_{L} \partial_{5} f_{R, n}^{a}(L) & =0 \\
g_{L} \partial_{5} f_{L, n}^{a}(L)-g_{R} \partial_{5} f_{R, n}^{a}(L) & =-\frac{1}{4}\left(g_{L}^{2}+g_{R}^{2}\right) a_{L}^{-2} L \tilde{v}^{2}\left(g_{L} f_{L, n}^{a}(L)-g_{R} f_{R, n}^{a}(L)\right) \\
\tilde{f}_{n}^{a} & =\frac{\sqrt{L} \tilde{v}}{2 m_{n}}\left(g_{L} f_{n, L}^{a}(L)-g_{R} f_{n, R}^{a}(L)\right)
\end{aligned}
$$

Finally, the Goldstone profiles are determined by the gauge profiles,

$$
\begin{array}{clrl}
\bar{f}_{n}\left(x_{5}\right) & =m_{n}^{-1} \partial_{5} f_{n}\left(x_{5}\right), & & \text { for } m_{n} \neq 0 \\
\bar{f}_{n} & =0, & & \text { for } m_{n}=0
\end{array}
$$

To calculate the explicit form of the profiles $f_{n}$, we solve the equation of motion such that it satisfies the conditions (2)-(4). Instead of solving it in a specific background it is more convenient to proceed in a background independent fashion. The equation of motion is second order so it has two independent solutions that correspond to warped trigonometric functions $C\left(x_{5}, m_{n}\right)$ and $S\left(x_{5}, m_{n}\right){ }^{2}$ We have freedom to choose them such that they

\footnotetext{
${ }^{1}$ The linear combination $B_{\mu}=s_{x} R_{\mu}^{3}+c_{x} X_{\mu}$ survives on the UV brane and its zero mode is identified with the hypercharge gauge boson. $B_{\mu}$ couples to matter with the coupling $g_{Y}=g_{X} g_{R} / \sqrt{g_{X}^{2}+g_{R}^{2}}$ and the hypercharge depends on the $\mathrm{SU}(2)_{R} \times \mathrm{U}(1)_{X}$ quantum numbers via $Y=t_{R}^{3}+X$.

${ }^{2}$ The properties of warped sines $S$ and cosines $C$ are discussed at more length in $\mathrm{A}$. In the flat space they are the well-known trigonometric functions, while in $\mathrm{AdS}_{\mathbf{5}}$ they can be expressed as combinations of Bessel and Neumann functions, see eq. (A.8).
} 
satisfy $C\left(0, m_{n}\right)=1, S^{\prime}\left(0, m_{n}\right)=m_{n}, C^{\prime}\left(0, m_{n}\right)=S\left(0, m_{n}\right)=0$. Then the profiles can be succinctly written:

$$
\begin{aligned}
& f_{L, n}^{a}\left(x_{5}\right)=\alpha_{L, n}^{a} C\left(x_{5}, m_{n}\right) \\
& f_{R, n}^{i}\left(x_{5}\right)=\alpha_{R, n}^{i} S\left(x_{5}, m_{n}\right) \\
& f_{R, n}^{3}\left(x_{5}\right)=\alpha_{N, n} s_{x} C\left(x_{5}, m_{n}\right)-\alpha_{D, n} c_{x} S\left(x_{5}, m_{n}\right) \\
& f_{X, n}\left(x_{5}\right)=\alpha_{N, n} c_{x} C\left(x_{5}, m_{n}\right)+\alpha_{D, n} s_{x} S\left(x_{5}, m_{n}\right)
\end{aligned}
$$

In this way, the profiles satisfy the UV boundary conditions (4.9). Inserting these expressions into the IR boundary conditions (4.10) we obtain relations between the normalization constants $\alpha_{n}$. We also obtain the quantization condition that factorizes as $F_{W}(m) F_{\gamma}(m) F_{Z}(m)=0$. This gives rise to three separate classes of solutions that we refer to as the $W, \gamma$ and $Z$ towers (because the lightest solution will be identified with the $\mathrm{W}$, the photon and the $\mathrm{Z}$, respectively). The quantization conditions for these three towers read:

$$
\begin{aligned}
\begin{array}{l}
0= \\
=
\end{array} & S^{\prime}\left(L, m_{W, n}\right) C^{\prime}\left(L, m_{W, n}\right) \\
& \quad+\frac{a_{L}^{-2} L \tilde{v}^{2}}{4}\left(g_{L}^{2} S^{\prime}\left(L, m_{W, n}\right) C\left(L, m_{W, n}\right)+g_{R}^{2} S\left(L, m_{W, n}\right) C^{\prime}\left(L, m_{W, n}\right)\right) \\
0= & C^{\prime}\left(L, m_{\gamma, n}\right) \\
0= & S^{\prime}\left(L, m_{Z, n}\right) C^{\prime}\left(L, m_{Z, n}\right) \\
& \quad+\frac{a_{L}^{-2} L \tilde{v}^{2}}{4}\left(g_{L}^{2} S^{\prime}\left(L, m_{Z, n}\right) C\left(L, m_{Z, n}\right)+g_{R}^{2} S\left(L, m_{Z, n}\right) C^{\prime}\left(L, m_{Z, n}\right)+m_{Z, n} a_{L}^{-2} g_{Y}^{2}\right)
\end{aligned}
$$

We give the exact profiles in B. In the main body of the paper we restrict ourselves to approximate expressions that are sufficient for our purposes.

\subsection{Scales}

From the quantization conditions (4.13)-(4.15) we can extract the mass spectrum. The model contains a tower of resonances starting at $\sim M_{\mathrm{KK}}$ where ${ }^{3}$

$$
M_{\mathrm{KK}}=\frac{\pi}{\int_{0}^{L} a^{-1}(y)} \sim \pi a^{\prime}(L)
$$

There are two exceptions. Firstly, as $\mathrm{U}(1)_{e m}$ is unbroken, there is always a massless vector boson - the photon. Secondly, there can be further states with masses parametrically below $M_{\mathrm{KK}}$ that are identified with the $\mathrm{W}$ and $\mathrm{Z}$ bosons. In eq. (A.5) we calculate their masses by expanding the warped trigonometric functions in eq. (4.13), (4.15) at small $\mathrm{m}$.

From these general results, we now consider two explicit limiting cases. First consider the limit where $\tilde{v} \gg M_{\mathrm{KK}}$. In this case the quantization conditions (4.13) and (4.15) are dominated by the second term. In such a limit we obtain

$$
m_{W}^{2} \approx \frac{g_{L}^{2} f_{h}^{2}}{4} \quad m_{Z}^{2} \approx \frac{\left(g_{L}^{2}+g_{Y}^{2}\right) f_{h}^{2}}{4}
$$

\footnotetext{
${ }^{3}$ The scale $M_{\mathrm{KK}}$ gives the parametric dependence of the mass of the lightest vector resonances. In $5 \mathrm{D}$ Minkowski the first KK photon mass is exactly equal to $M_{\mathrm{KK}}$, while in $\mathrm{AdS}_{\mathbf{5}}$ it is approximately $0.8 M_{\mathrm{KK}}$.
} 
where the scale $f_{h}$, called the Higgs decay constant, is fixed by the geometry of the 5th dimension:

$$
f_{h}^{2}=\frac{4}{L\left(g_{L}^{2}+g_{R}^{2}\right) \int_{0}^{L} a^{-2}(y)}
$$

Thus in this limit $v \approx f_{h}$ and, as we will see later, $g_{h}=0$. Therefore the Higgs plays no role in unitarising the gauge boson scattering, though it remains in the spectrum. ${ }^{4} \mathrm{We}$ refer to this limit as the Higgsless limit.

If we take $g_{L} \sim g_{R}$, we get:

$$
g_{L}^{2} f_{h}^{2} \sim \frac{4 M_{\mathrm{KK}}^{2}}{\pi^{2} \mathcal{V}} \quad \mathcal{V} \equiv \frac{L a^{\prime}(L)}{a_{L}}
$$

We have introduced the geometric factor $\mathcal{V}$ that we call the volume factor. In the Higgsless limit we need the volume factor to be large, otherwise there is no separation between $v$ and $M_{\mathrm{KK}}$. Such a case is ruled out by searches for light resonances. In flat space we get $\mathcal{V}=1$. In contrast, within the Randall-Sundrum $\mathrm{AdS}_{\mathbf{5}}$ setup it is $\mathcal{V}=k L \sim$ $\log \left(M_{\mathrm{Pl}} / M_{\mathrm{KK}}\right) \sim 30$. As discussed in [12], we expect $\mathcal{V}$ to be large if the $5 \mathrm{D}$ set-up is to solve the hierarchy problem.

The volume factor could be made arbitrarily large by an educated choice of the $5 \mathrm{D}$ geometry. Note however that consistency arguments set an upper bound, $\mathcal{V} \lesssim 16 \pi$. Otherwise, the resonance scale would be pushed above $1 \mathrm{TeV}$ and the gauge boson scattering would get strong before the vector resonances set in to restore unitarity.

Now consider the opposite limit in which $\tilde{v} \ll M_{\mathrm{KK}} \cdot{ }^{5}$ In this case $g_{h} \approx 1$ and the Higgs boson is SM-like. We refer to this limit as the Higgs limit. Once again, this allows us to obtain the desired scale separation $v \ll M_{\mathrm{KK}}$. For $\tilde{v} / M_{\mathrm{KK}} \ll 1$ the electroweak gauge boson masses are approximately given by

$$
m_{W}^{2} \approx \frac{g_{L}^{2} \tilde{v}^{2}}{4} \quad m_{Z}^{2} \approx \frac{g_{L}^{2} \tilde{v}^{2}}{4 \cos ^{2} \theta_{W}}
$$

Thus, the electroweak scale in the Higgs limit is simply $v \approx \tilde{v}$. More precisely, the relation between the two scales is of the form $v^{2} \approx \tilde{v}^{2}\left(1-\frac{\tilde{v}^{2}}{f_{h}^{2}}\right)$. The scale $f_{h}$ that appears in this relation is the one defined by eq. (4.18). Although in the Higgs limit $f_{h}$ enters in a rather intricate way, it will turn out to play an important role in describing the $W$ and $Z$ scattering.

Summarizing, the following three scales have emerged: the electroweak scale $v$, the Higgs decay constant $f_{h}$ and the resonance scale $M_{\mathrm{KK}}$. The separation between $f_{h}$ and $M_{\mathrm{KK}}$ is set by a geometric quantity we call the volume factor. In the Higgs limit the electroweak scale can be adjusted to be smaller than $f_{h}$, while in the Higgsless limit $v$ and $f_{h}$ coincide.

\footnotetext{
${ }^{4}$ Generically, in this limit we expect the Higgs boson to be heavy, $m_{h} \sim \tilde{v}$, but if its self-coupling is very weak it may remain light.

${ }^{5}$ The condition $\tilde{v} \ll M_{\mathrm{KK}}$ is a postulate, but in general this input is a consequence of some unspecified dynamics that gives rises to the boundary Higgs potential. We take $\tilde{v} / M_{\mathrm{KK}}$ as a free parameter and set it to be small but getting to generate $\tilde{v} / M_{\mathrm{KK}}<1 / 4 \pi$ would typically require fine-tuning.
} 


\subsection{Goldstone bosons}

The important input for calculating gauge boson scattering amplitudes are the profiles of the Goldstone bosons $G^{+}, G^{-}$and $G^{3}$ that are eaten by the physical $W^{+}, W^{-}$and $Z$ bosons respectively. In general, they are linear combinations of $L_{5}, R_{5}, X_{5}$ and $\tilde{G}$. The exact profiles are given in eq. (B.5). To leading order in $m_{W} / M_{\mathrm{KK}}$, these profiles can be concisely described in a background independent way. With the help of eq. (A.5) we find

$$
\begin{aligned}
\bar{f}_{L, W}^{i} & \approx-\frac{1}{\sqrt{L}} m_{W} x_{5} a^{-2}\left(x_{5}\right) \\
\bar{f}_{R, W}^{i} & \approx \frac{1}{\sqrt{L}} \frac{g_{R}}{g_{L}} m_{W} L a^{-2}\left(x_{5}\right) \\
\tilde{f}_{W}^{i} & \approx \frac{v}{\tilde{v}} \\
\bar{f}_{L, Z}^{3} & \approx-\frac{1}{\sqrt{L}} m_{W} x_{5} a^{-2}\left(x_{5}\right) \\
\bar{f}_{R, Z}^{3} & \approx \frac{1}{\sqrt{L}} \frac{g_{R}}{g_{L}}\left(s_{x}^{2} x_{5}+c_{x}^{2} L\right) m_{W} a^{-2}\left(x_{5}\right) \\
\bar{f}_{X, Z} & \approx c_{x} \frac{\tan \theta_{W}}{\sqrt{L}}\left(x_{5}-L\right) m_{W} a^{-2}\left(x_{5}\right) \\
\tilde{f}_{Z}^{3} & \approx \frac{v}{\tilde{v}}
\end{aligned}
$$

In the Higgs limit, $v \approx \tilde{v}$ and the Goldstones reside mostly on the brane. In the Higgsless limit, $\tilde{v} \gg v$ and the Goldstones live mostly in the bulk, though for warped metrics they are still sharply localized at the IR brane due to the $a^{-2}\left(x_{5}\right)$ profile.

With this information, we can read off the quartic self-couplings of the Goldstones and the Higgs-Goldstone couplings. We find

$$
\begin{aligned}
\mathcal{L}_{G^{4}} & =-\frac{g_{h}^{2}}{6 v^{2}}\left(\partial_{\mu} G^{a}\right)^{2} G^{2}+\frac{g_{h}^{2}}{6 v^{2}}\left(\partial_{\mu} G^{a} G^{a}\right)^{2} \\
\mathcal{L}_{G^{2} h} & =\frac{g_{h}}{v} h\left(\partial_{\mu} G^{a}\right)^{2}
\end{aligned}
$$

where $g_{h}=v^{3} / \tilde{v}^{3}$. Thus, $g_{h} \approx 0$ in the Higgsless limit, while $g_{h} \approx 1$ in the Higgs limit, as indicated before.

\subsection{Couplings of resonances}

To calculate the total gauge boson scattering amplitude we need to calculate the coupling of the Goldstone bosons to the resonances. The Goldstones interact with the charged and neutral resonances via the triple vertices:

$$
\begin{aligned}
\mathcal{L}_{G^{2} A}= & -i\left(\partial_{\mu} G^{+} G^{-}-\partial_{\mu} G^{-} G^{+}\right) g_{N, n} A_{\mu, n} \\
& +\left\{-i\left(\partial_{\mu} G^{-} G^{3}-\partial_{\mu} G^{3} G^{-}\right) g_{C, n} W_{\mu, n}^{+}+\text {h.c. }\right\}
\end{aligned}
$$

where $N$ stands for neutral bosons $\gamma, Z$ and $C$ stands for the charged $W$. The resonance couplings $g_{n}$ can be found by inserting the Goldstone and resonance profiles into the interaction terms in the $5 \mathrm{D}$ action. In order to somewhat simplify the resulting expressions we 
set $g_{L}=g_{R}$. Furthermore, the Goldstone profiles are localized toward the IR brane. This results in the factor $a^{-2}(y)$ showing up in the integrals. Therefore the integrals are dominated by the IR region and it is a good approximation to replace $y \approx L$ in the integrands. This allows us to approximate the resonance couplings as

$$
\begin{array}{r}
g_{N, n} \approx g_{L} m_{W}^{2} L^{3 / 2} \int_{0}^{L} a^{-2}(y)\left\{f_{L, n}^{3}(y)+f_{R, n}^{3}(y)\right\} \\
+\frac{1}{2} \sqrt{L} g_{L} \frac{v^{2}}{\tilde{v}^{2}}\left(f_{L, n}^{3}(L)+f_{R, n}^{3}(L)\right) \\
g_{W, n} \approx g_{L} m_{W}^{2} L^{3 / 2} \int_{0}^{L} a^{-2}(y)\left\{f_{L, n}^{i}(y)+f_{R, n}^{i}(y)\right\} \\
+\frac{1}{2} \sqrt{L} g_{L} \frac{v^{2}}{\tilde{v}^{2}}\left(f_{L, n}^{i}(L)+f_{R, n}^{i}(L)\right)
\end{array}
$$

The first term within the integral dominates the Higgsless limit, $v \ll \tilde{v}$, when the Goldstones live in the bulk. The second term dominates the Higgs limit, when the Goldstones live on the brane. For $n$ corresponding to the electroweak gauge bosons we recover the Standard Model couplings $g_{0}: g_{W} \approx \frac{g_{L}}{2}, g_{Z} \approx \frac{g_{L}^{2}-g_{Y}^{2}}{2 \sqrt{g_{L}^{2}+g_{Y}^{2}}}, g_{\gamma}=e$. For $n$ corresponding the heavy resonances the results are collected in eq. (B.9).

In order to estimate the resonance couplings we can employ the approximation (A.6) to the profiles of resonances. The simple pattern that emerges is that all resonance couplings, $g_{n}$, are parametrically enhanced with respect to the SM ones by a common factor:

$$
g_{n} \sim \sqrt{\mathcal{V}} g_{0}
$$

where $\mathcal{V}$ is the volume factor defined in eq. (4.19) and $g_{0}$ is the SM coupling relevant for the given tower: $g_{W}, g_{Z}$ or $g_{\gamma}$. Since $\mathcal{V} \sim M_{\mathrm{KK}}^{2} / f_{h}^{2}$, we get $f_{h} g_{n} \sim M_{\mathrm{KK}} g_{0}$. For $g_{0} \sim 1$ this coincides with the relation advertised in eq. (1) of ref. [0].

To make the discussion more quantitative, consider first the couplings of the charged resonances in the Higgs limit. In this case the quantization condition for the $W$ tower (4.13) reduces to $C^{\prime}\left(L, m_{W, n}\right) S^{\prime}\left(L, m_{W, n}\right) \approx 0$. Thus, the mass eigenstates in the $\mathrm{W}$ tower split into Neumann-Neumann (NN) and Dirichlet-Neumann (DN) modes (B.1):

$$
\begin{array}{ll}
(\mathrm{NN}): \quad f_{L, n}^{i} \approx \frac{C\left(x_{5}, m_{W, n}\right)}{\left(\int_{0}^{L}\left[C\left(y, m_{W, n}\right)\right]^{2}\right)^{1 / 2}} & C^{\prime}\left(L, m_{W, n}\right)=0 \\
(\mathrm{DN}): & f_{R, n}^{i} \approx \frac{S\left(x_{5}, m_{W, n}\right)}{\left(\int_{0}^{L}\left[S\left(y, m_{W, n}\right)\right]^{2}\right)^{1 / 2}} \quad S^{\prime}\left(L, m_{W, n}\right)=0
\end{array}
$$

For warped metrics, the UV boundary conditions are not relevant for the behaviour of the resonance profiles in IR (they only affect the light modes that are delocalized). More precisely, we have the relations

$$
C(L, m) \approx-m L S(L, m) \quad C^{\prime}(L, m) \approx-m L S^{\prime}(L, m)
$$


that hold for $m \sim M_{\mathrm{KK}}$. In consequence, the NN and the DN modes have approximately the same masses and, from eq. (4.27), couple with approximately the same strength to the electroweak Goldstone bosons. Thus, at the scale of the first resonance the Goldstone bosons couple to two almost degenerate charged vector states. Moreover, using the methods of [14], one can prove some remarkable sum rules. A profile satisfying the DN boundary conditions has the integral representation $f_{n}(y)=m_{n}^{2} \int_{0}^{y} a^{-2} \int_{y^{\prime}}^{L} f_{n}\left(y^{\prime \prime}\right)$. Thus

$$
\sum_{n} \frac{f_{n}(L)^{2}}{m_{n}^{2}}=\sum_{n} f_{n}(L) \int_{0}^{L} a^{-2} \int_{y^{\prime}}^{L} f_{n}\left(y^{\prime \prime}\right)=\int_{0}^{L} a^{-2}
$$

where we have used the completeness relation $\sum_{n} f_{n}(x) f_{n}(y)=\delta(x-y)$. For NN modes we get approximately the same sum rule, once the contribution of the SM $W$ boson is omitted in the sum. Using these results we easily obtain

$$
\sum_{n>0} \frac{g_{W, n}^{2}}{m_{W, n}^{2}} \approx \frac{1}{f_{h}^{2}}
$$

This sum rule is typically dominated by the first two terms, so we find

$$
g_{W, 1}^{2} \approx g_{W, 2}^{2} \approx \frac{m_{W, 1}^{2}}{2 f_{h}^{2}}
$$

This is in accord with the parametric estimate (4.28).

The Higgsless limit, although qualitatively similar, differs in several details. The $W$ tower splits now into the vector and axial modes:

$$
\begin{aligned}
& f_{L, n}^{i} \approx \frac{C\left(x_{5}, m_{W, n}\right)}{\left(2 \int_{0}^{L}\left[C\left(y, m_{W, n}\right)\right]^{2}\right)^{1 / 2}} \quad f_{R, n}^{i} \approx f_{L, n}^{i} \quad C^{\prime}\left(L, m_{W, n}\right)=0 \\
& f_{L, n}^{i} \approx \frac{C\left(x_{5}, m_{W, n}\right)}{\left(2 \int_{0}^{L}\left[C\left(y, m_{W, n}\right)\right]^{2}\right)^{1 / 2}} \quad f_{R, n}^{i} \approx-f_{L, n}^{i} \quad S\left(L, m_{W, n}\right)=0
\end{aligned}
$$

From eq. (4.27), only the vector modes couple to the electroweak Goldstones. The sum rule now becomes

$$
\sum_{n>0} \frac{g_{W, n}^{2}}{m_{W, n}^{2}} \approx \frac{1}{3 f_{h}^{2}}
$$

At the scale of the first resonance only one vector state appears in the Goldstone scattering amplitude. Its coupling can be estimated as

$$
g_{W, 1}^{2} \approx \frac{m_{W, 1}^{2}}{3 f_{h}^{2}},
$$

so that it is slightly weaker than in the Higgs limit.

To complete the picture let us discuss the cutoff scale where the 5D theory becomes strongly coupled. From the parametric dependence of the resonance couplings we conclude the cutoff is fixed by the volume factor. We can estimate:

$$
\Lambda \sim \frac{4 \pi}{g_{0}^{2} \mathcal{V}} M_{\mathrm{KK}}
$$


We see the volume factor should not be larger than $\sim 16 \pi$. Otherwise, the first resonance would already be strongly coupled and the $5 \mathrm{D}$ description would not be meaningful.

\section{Holographic pseudo-Goldstone Higgs}

We move to another, closely related higher-dimensional setup. We consider a $5 \mathrm{D} \mathrm{SU}(3)_{C} \times$ $\mathrm{SO}(5) \times \mathrm{U}(1)_{X}$ gauge theory broken to $\mathrm{SU}(3)_{C} \times \mathrm{SU}(2)_{L} \times \mathrm{U}(1)_{Y}$ on the UV brane, and to $\mathrm{SU}(3)_{C} \times \mathrm{SO}(4) \times \mathrm{U}(1)_{X}$ on the IR brane [5]. The larger symmetry group of the bulk allows us to accommodate the Higgs field as the 5th component of the gauge bosons. The Higgs field is massless at tree level due to $5 \mathrm{D}$ gauge invariance, but it acquires a potential at one loop. Thus, the origin of the light Higgs field is addressed in this set-up (rather than postulated, as in the previous one). In a fully fledged theory the Higgs field would acquire a vev dynamically through minimization of the Coleman-Weinberg potential. Here however, we will not study the dynamics that produces the vev, but simply assume it exists. Holographically, this setup again corresponds to the Standard Model coupled to a strongly interacting sector that breaks electroweak symmetry. The global symmetry of the strong sector is $\mathrm{SU}(3)_{C} \times \mathrm{SO}(5) \times \mathrm{U}(1)_{X} . \mathrm{SO}(5)$ is spontaneously broken to $\mathrm{SO}(4)$ by the strong dynamics. The resulting pseudo-Goldstones are identified with the Higgs field.

We concentrate on the $\mathrm{SO}(5) \times \mathrm{U}(1)_{X}$ part with the gauge fields $A_{M}=A_{M}^{\alpha} T^{\alpha}$ and $X_{M}$. The dimensionful bulk gauge couplings are denoted as $g \sqrt{L}$ and $g_{X} \sqrt{L}$. The $5 \mathrm{D}$ action is

$$
S_{5 \mathrm{D}}=\int d^{4} x \int_{0}^{L} d x_{5} \sqrt{g}\left(-\frac{1}{4} \operatorname{Tr}\left\{A_{M N} A^{M N}\right\}-\frac{1}{4} X_{M N} X^{M N}\right),
$$

\subsection{Mass eigenstates}

We employ the mass eigenstate formalism for the KK expansion. We want to arrive at the quadratic action of the form (4.6) which is diagonal in the KK index in the presence of electroweak symmetry breaking. In contrast to the previous section there is an added complication of the $A_{5} \mathrm{vev}$ which affects the quadratic terms in the action. The changes can, however, be simply taken into account by replacing $\partial_{5}$ with the covariant derivative $D_{5}=\partial_{5}-i g \sqrt{L}\left[\left\langle A_{5}\right\rangle, \cdot\right]$.

We perform the KK decomposition, in the presence of the $A_{5}$ vev:

$$
\begin{array}{ll}
A_{\mu}\left(x, x_{5}\right)=A_{\mu, n}(x) f_{n}\left(x_{5},\left\langle A_{5}\right\rangle\right) & A_{5}\left(x, x_{5}\right)=\left\langle A_{5}\left(x_{5}\right)\right\rangle+G_{n}(x) \bar{f}_{n}\left(x_{5},\left\langle A_{5}\right\rangle\right) \\
X_{\mu}\left(x, x_{5}\right)=A_{\mu, n}(x) f_{X, n}\left(x_{5},\left\langle A_{5}\right\rangle\right) & X_{5}\left(x, x_{5}\right)=G_{n}(x) \bar{f}_{X, n}\left(x_{5},\left\langle A_{5}\right\rangle\right)
\end{array}
$$

where $f_{n}=f_{n}^{\alpha} T^{\alpha}$. We split the $\mathrm{SO}(5)$ generators as $T^{\alpha}=\left(T_{L}^{a}, T_{R}^{a}, T_{C}^{\hat{a}}\right), a=1 \ldots 3$, $\hat{a}=1 \ldots 4$, corresponding to $\mathrm{SU}(2)_{L}$ and $\mathrm{SU}(2)_{R}$ subgroups and the $\mathrm{SO}(5) / \mathrm{SO}(4)$ coset. Accordingly, we also split the gauge field $A_{M}=\left(L_{M}, R_{M}, C_{M}\right)$ and the profiles $f_{n}=$ $\left(f_{L, n}, f_{R, n}, f_{C, n}\right)$.

Diagonalization is achieved when the profiles satisfy the following conditions:

1. The equation of motion in the $A_{5}$ background:

$$
D_{5}\left(a^{2} D_{5} f_{n}\right)+m_{n}^{2} f_{n}=0
$$




$$
D_{5} f_{n}=\partial_{5} f_{n}-i g \sqrt{L}\left[\left\langle A_{5}\left(x_{5}\right)\right\rangle, f_{n}\right]
$$

$f_{n}^{X}$ satisfies the same equation with $D_{5} \rightarrow \partial_{5}$.

2. The normalization condition:

$$
\int_{0}^{L}\left\{\operatorname{Tr}\left[f_{n}\left(y,\left\langle A_{5}\right\rangle\right) f_{n}\left(y,\left\langle A_{5}\right\rangle\right)\right]+f_{n}^{X}\left(y,\left\langle A_{5}\right\rangle\right) f_{n}^{X}\left(y,\left\langle A_{5}\right\rangle\right)\right\}=1
$$

3. IR boundary conditions:

$$
\begin{aligned}
f_{C, n}^{\hat{a}}\left(L,\left\langle A_{5}\right\rangle\right) & =0 \\
D_{5} f_{L, n}^{a}\left(L,\left\langle A_{5}\right\rangle\right) & =0 \\
D_{5} f_{R, n}^{a}\left(L,\left\langle A_{5}\right\rangle\right) & =0 \\
\partial_{5} f_{X, n}\left(L,\left\langle A_{5}\right\rangle\right) & =0
\end{aligned}
$$

that break $\mathrm{SO}(5) \times \mathrm{U}(1)_{X}$ to $\mathrm{SO}(4) \times \mathrm{U}(1)_{X}$.

4. UV boundary conditions:

$$
\begin{array}{rlrl}
\partial_{5} f_{L, n}^{a}\left(0,\left\langle A_{5}\right\rangle\right) & =0 & & \\
f_{R, n}^{i}\left(0,\left\langle A_{5}\right\rangle\right) & =0 & & i=1,2 \\
s_{x} \partial_{5} f_{R, n}^{3}\left(0,\left\langle A_{5}\right\rangle\right)+c_{x} \partial_{5} f_{X, n}\left(0,\left\langle A_{5}\right\rangle\right) & =0 & & s_{x}=\frac{g_{X}}{\sqrt{g_{X}^{2}+g_{L}^{2}}} \\
-c_{x} f_{R, n}^{3}\left(0,\left\langle A_{5}\right\rangle\right)+s_{x} f_{X, n}\left(0,\left\langle A_{5}\right\rangle\right) & =0 & & c_{x}=\frac{g_{L}}{\sqrt{g_{X}^{2}+g_{L}^{2}}} \\
f_{C, n}^{\hat{a}}\left(0,\left\langle A_{5}\right\rangle\right) & =0 & \hat{a}=1 \ldots 4
\end{array}
$$

that break $\mathrm{SO}(5) \times \mathrm{U}(1)_{X}$ to $\mathrm{SU}(2)_{L} \times \mathrm{U}(1)_{Y}$, the hypercharge being a linear combination of $\mathrm{SU}(2)_{R} \times \mathrm{U}(1)_{X}$. The SM gauge couplings are $g_{L}=g$ and $g_{Y}=\frac{g_{X} g}{\sqrt{g_{X}^{2}+g^{2}}}$

The Goldstone profiles are chosen accordingly:

$$
\bar{f}_{n}\left(x_{5},\left\langle A_{5}\right\rangle\right)=m_{n}^{-1} D_{5} f_{n}\left(x_{5},\left\langle A_{5}\right\rangle\right)
$$

In general, the profiles in the $A_{5}$ background are related to the profiles at $\left\langle A_{5}\right\rangle=0$ by a rotation via the Wilson-line matrix,

$$
\begin{aligned}
f_{n}\left(x_{5},\left\langle A_{5}\right\rangle\right) & =\omega^{-1}\left(x_{5},\left\langle A_{5}\right\rangle\right) f_{n}\left(x_{5}\right) \omega\left(x_{5},\left\langle A_{5}\right\rangle\right), \\
\text { where } \omega & =P \exp \left(-i g \sqrt{L} T^{\alpha} \int_{0}^{x_{5}}\left\langle A_{5}^{\alpha}\right\rangle\right)
\end{aligned}
$$

and $f_{n}^{X}\left(x_{5},\left\langle A_{5}\right\rangle\right)=f_{n}^{X}\left(x_{5}\right)$. The profiles $f_{n}\left(x_{5}\right)$ satisfy the "normal" equation of motion, $\partial_{5}\left(a^{2} \partial_{5} f_{n}\right)+m_{n}^{2} f_{n}=0$. Similarly, for the Goldstone profiles

$$
\bar{f}_{n}\left(x_{5},\left\langle A_{5}\right\rangle\right)=m_{n}^{-1} \omega^{-1}\left(x_{5},\left\langle A_{5}\right\rangle\right) \partial_{5} f_{n}\left(x_{5}\right) \omega\left(x_{5},\left\langle A_{5}\right\rangle\right)
$$


In the following we choose the basis such that the vev resides in only one direction in the group space:

$$
\left\langle A_{5}^{\hat{4}}\right\rangle=\frac{a^{-2}\left(x_{5}\right)}{\sqrt{\int_{0}^{L} a^{-2}(y)}} \tilde{v}
$$

The profiles at zero vev can be written as

$$
\begin{aligned}
& f_{L, n}^{a}\left(x_{5}\right)=\alpha_{L}^{a} C\left(x_{5}, m_{n}\right) \\
& f_{R, n}^{i}\left(x_{5}\right)=\alpha_{R}^{i} S\left(x_{5}, m_{n}\right) \quad i=1,2 \\
& f_{R, n}^{3}\left(x_{5}\right)=\alpha_{N} s_{x} C\left(x_{5}, m_{n}\right)-\alpha_{D} c_{x} S\left(x_{5}, m_{n}\right) \\
& f_{X, n}\left(x_{5}\right)=\alpha_{N} c_{x} C\left(x_{5}, m_{n}\right)+\alpha_{D} s_{x} S\left(x_{5}, m_{n}\right) \\
& f_{C, n}^{\hat{a}}\left(x_{5}\right)=\alpha_{C}^{\hat{a}} S\left(x_{5}, m_{n}\right)
\end{aligned}
$$

They satisfy the UV boundary conditions (5.6). The constants are obtained by imposing the IR boundary conditions. The solutions can be organized into two towers $W_{n}, \bar{W}_{n}$ of charged gauge bosons, and four towers $\gamma_{n}, Z_{n}, \bar{Z}_{n}, H_{n}$ of neutral ones. We list all the profiles in C. Here we content ourselves with the quantization conditions:

$$
\begin{array}{rlrl}
C^{\prime}\left(L, m_{W, n}\right) S\left(L, m_{W, n}\right)+\frac{1}{2} m_{W, n} a_{L}^{-2} \sin ^{2}\left(\frac{\tilde{v}}{f_{h}}\right) & =0 & S^{\prime}\left(L, m_{\bar{W}, n}\right) & =0 \\
\cos ^{2} \theta_{W} C^{\prime}\left(L, m_{Z, n}\right) S\left(L, m_{Z, n}\right)+\frac{1}{2} m_{Z, n} a_{L}^{-2} \sin ^{2}\left(\frac{\tilde{v}}{f_{h}}\right) & =0 & S^{\prime}\left(L, m_{\bar{Z}, n}\right) & =0 \\
C^{\prime}\left(L, m_{\gamma, n}\right) & =0 & S\left(L, m_{H, n}\right) & =0
\end{array}
$$

Only the masses in the $W$ and $Z$ towers are sensitive to electroweak symmetry breaking. The scale $f_{h}$ is once again defined in terms of geometric quantities:

$$
f_{h}^{2}=\frac{2}{g_{L}^{2} L \int_{0}^{L} a^{-2}(y)}
$$

This definition coincides with eq. (4.18), once $g_{L}=g_{R}$. In the present model $f_{h}$ appears as the symmetry breaking scale at which the global $\mathrm{SO}(5)$ is broken to $\mathrm{SO}(4)$. Its role in the $W$ and $Z$ scattering will turn out analogous as in the previous model.

\subsection{Scales}

The tower of heavy resonances begins at $\sim M_{\mathrm{KK}}$ (defined in eq. (4.16)). In addition the setup can accommodate light vector states identified with the electroweak gauge bosons. There is always the photon with $m_{\gamma}=0$. The lightest massive vector states in the $W$ and $Z$ tower are identified with the $W$ and $Z$ bosons and can be parametrically lighter than $M_{\mathrm{KK}}$. Finally, there are no light states in the remaining towers. Expanding the warped trigs in eq. (5.13) and eq. (5.14) for small masses we find the $W$ and $Z$ masses:

$$
m_{W}^{2} \approx \frac{g_{L}^{2}}{4} f_{h}^{2} \sin ^{2}\left(\frac{\tilde{v}}{f_{h}}\right)
$$




$$
m_{Z}^{2} \approx \frac{g_{L}^{2}+g_{Y}^{2}}{4} f_{h}^{2} \sin ^{2}\left(\frac{\tilde{v}}{f_{h}}\right)
$$

Thus we identify the electroweak scale as $v \approx f_{h} \sin \left(\tilde{v} / f_{h}\right)$. The separation between the electroweak scale and the resonance scale can be achieved in two separate limits. In the first case we assume a separation between the Higgs vev and the decay constant, $\sin \left(\tilde{v} / f_{h}\right) \ll 1$. This is the Higgs limit. $\sin \left(\tilde{v} / f_{h}\right)$ is a free parameter until we specify the dynamics that gives rises to the Higgs potential. One should be aware, however, that getting $\tilde{v} / f_{h}$ smaller than 1 typically requires fine-tuning. In the other limit, $\sin \left(\tilde{v} / f_{h}\right) \sim 1$. This corresponds to the Higgsless limit. Once again we need to separate $f_{h}$ from $M_{\mathrm{KK}}$. The separation $f_{h} \ll M_{\mathrm{KK}}$ is obtained in the 5D background with a large volume factor $\mathcal{V}$.

Summarizing, the three scales $v, f_{h}$, and $M_{\mathrm{KK}}$ have emerged again. In the Higgsless limit $v \approx f_{h}$. In fact, in this limit the quantization conditions for the $W$ and $Z$ tower masses are exactly the same as in the previous model. The physics in the Higgsless limit is indistinguishable in these two models.

\subsection{Goldstone bosons}

To calculate the gauge boson scattering amplitudes we need to calculate the Goldstone boson profiles corresponding to $W, Z$. The exact profiles are written in eq. (C.15). Expanding these profiles in powers of $m_{W}^{2}$ and $m_{Z}^{2}$ we find, at lowest order:

$$
\begin{aligned}
\bar{f}_{L, W}^{i} & \approx-\frac{1}{\sqrt{L}} m_{W} x_{5} a^{-2}\left(x_{5}\right) \\
\bar{f}_{R, W}^{i} & \approx \frac{1}{\sqrt{L}} m_{W} L a^{-2}\left(x_{5}\right) \\
\bar{f}_{C, W}^{i} & \approx \frac{1}{\sqrt{L}} \frac{\sqrt{2} \cos \left(\tilde{v} / f_{h}\right)}{\sin \left(\tilde{v} / f_{h}\right)} m_{W} L a^{-2}\left(x_{5}\right) \\
\bar{f}_{L, Z}^{3} & \approx-\frac{1}{\sqrt{L}} m_{W} x_{5} a^{-2}\left(x_{5}\right) \\
\bar{f}_{R, Z}^{3} & \approx \frac{1}{\sqrt{L}}\left(s_{x}^{2} x_{5}+c_{x}^{2} L\right) m_{W} a^{-2}\left(x_{5}\right) \\
\bar{f}_{X, Z} & \approx c_{x} \frac{\tan \theta_{W}}{\sqrt{L}}\left(x_{5}-L\right) m_{W} a^{-2}\left(x_{5}\right) \\
\bar{f}_{C, Z}^{3} & \approx \frac{1}{\sqrt{L}} \frac{\sqrt{2} \cos \left(\tilde{v} / f_{h}\right)}{\sin \left(\tilde{v} / f_{h}\right)} L m_{W} a^{-2}\left(x_{5}\right)
\end{aligned}
$$

Compared to the previous model, there are no boundary Goldstones. Instead their role is taken over by $C_{5}^{a}$. The parameter controlling the distribution of the Goldstones is now $\sin \left(\tilde{v} / f_{h}\right)$. In the Higgs limit the electroweak Goldstone bosons are mainly composed of $C_{5}^{a}$. In the Higgsless limit the electroweak Goldstones flow to $L_{5}^{a}$ and $R_{5}^{a}$. In all cases the Goldstones are sharply localized on the IR brane with the profile behaving as $a^{-2}$. The self-interactions of the non-linearly defined Goldstones and the triple vertex with the Higgs 
boson are described by eq. (4.24) with $g_{h}=\cos \left(\tilde{v} / f_{h}\right) .{ }^{6}$ In the Higgsless limit the physical Higgs decouples from the Goldstones but it remains in the physical spectrum.

\subsection{Couplings to resonances}

The couplings of the electroweak Goldstone bosons to the resonances are given in $\mathrm{q}$. In the following discussion we again make the assumption that the warp factor is steep enough close to the IR brane, so that we can replace $y \rightarrow L$ in all integrals. In such a case, the coupling of the vertices defined in eq. 4.25 can be written as

$$
\begin{aligned}
& g_{C, n} \approx g_{L} L^{3 / 2} m_{W}^{2} \int_{0}^{L} a^{-2}\left\{f_{L, n}^{i}+f_{R, n}^{i}\right\} \\
& g_{N, n} \approx g_{L} L^{3 / 2} m_{W}^{2} \int_{0}^{L} a^{-2}\left\{f_{L, n}^{3}+f_{R, n}^{3}\right\}
\end{aligned}
$$

where $C=W, \bar{W}$ stands for charged, while $N=Z, \bar{Z}, \gamma$ stands for neutral (the vector bosons from the $H$ tower do not couple to the electroweak Goldstones). The Standard Model gauge bosons couple to the electroweak gauge bosons as $g_{W} \approx g_{L} / 2, g_{Z} \approx \frac{g_{L}^{2}-g_{Y}^{2}}{2 \sqrt{g_{L}^{2}+g_{Y}^{2}}}$, $g_{\gamma}=e$. The resonance couplings depend on their profiles which we collected in G. Parametrically, we again observe an enhancement of the resonance couplings

$$
g_{n} \sim \sqrt{\mathcal{V}} g_{0}
$$

where $g_{0}$ is the coupling of the corresponding Standard Model gauge boson. Moreover, the couplings of the $\bar{W}$ and $\bar{Z}$ towers are also proportional to $\cos \left(\tilde{v} / f_{h}\right)$, so that they decouple from the electroweak Goldstones in the Higgsless limit.

Consider first the couplings of the charged resonances in the Higgs limit. In this case the quantization condition in the $W$ tower (5.13) reduces to $C^{\prime}\left(L, m_{W, n}\right) S\left(L, m_{W, n}\right) \approx 0$. Thus, the mass eigenstates in the $W$ tower split into Neumann-Neumann (NN) and DirichletDirichlet (DD) modes:

$$
\begin{array}{ll}
(\mathrm{NN}): f_{L, n}^{i} \approx \frac{C\left(x_{5}, m_{W, n}\right)}{\left(\int_{0}^{L}\left[C\left(y, m_{W, n}\right)\right]^{2}\right)^{1 / 2}} & C^{\prime}\left(L, m_{W, n}\right)=0 \\
(\mathrm{DD}): f_{C, n}^{i} \approx \frac{S\left(x_{5}, m_{W, n}\right)}{\left(\int_{0}^{L}\left[S\left(y, m_{W, n}\right)\right]^{2}\right)^{1 / 2}} & S\left(L, m_{W, n}\right)=0
\end{array}
$$

From eq. (5.21), only the NN modes couple to the electroweak Goldstone bosons. The $\bar{W}$ tower has the profile of the DN type

$$
(\mathrm{DN}): f_{R, n}^{i} \approx \frac{S\left(x_{5}, m_{\bar{W}, n}\right)}{\left(\int_{0}^{L}\left[S\left(y, m_{\bar{W}, n}\right)\right]^{2}\right)^{1 / 2}} \quad S^{\prime}\left(L, m_{\bar{W}, n}\right)=0
$$

As discussed before the $\mathrm{NN}$ and the DN resonances have approximately the same profiles and masses, therefore they couple with approximately the same strength to the electroweak

\footnotetext{
${ }^{6}$ Suppression of the WWh and ZZh vertices by $\cos \left(\tilde{v} / f_{h}\right)$ was also pointed out in ref. 15.
} 
Goldstone bosons. Thus, at the scale of the first resonance, there are two degenerate charged vector states. Moreover we obtain the following sum rules:

$$
\sum_{n} \frac{g_{W, n}^{2}}{m_{W, n}^{2}} \approx \sum_{n} \frac{g_{\bar{W}, n}^{2}}{m_{\bar{W}, n}^{2}} \approx \frac{1}{6 f_{h}^{2}}
$$

The sum rules are typically dominated by the first term, so we get

$$
g_{W, 1}^{2} \approx g_{\bar{W}, 1}^{2} \approx \frac{m_{W, 1}^{2}}{6 f_{h}^{2}}
$$

With respect to gauge boson scattering, the Higgsless limit is indistinguishable from the previous model. The $\bar{W}$ tower decouples from the electroweak Goldstone bosons. The $W$ tower splits now into the vector and axial modes:

$$
\begin{aligned}
& f_{L, n}^{i} \approx \frac{C\left(x_{5}, m_{W, n}\right)}{\left(2 \int_{0}^{L}\left[C\left(y, m_{W, n}\right)\right]^{2}\right)^{1 / 2}} \quad f_{R, n}^{i} \approx f_{L, n}^{i} \quad C^{\prime}\left(L, m_{W, n}\right)=0 \\
& f_{L, n}^{i} \approx \frac{C\left(x_{5}, m_{W, n}\right)}{\left(2 \int_{0}^{L}\left[C\left(y, m_{W, n}\right)\right]^{2}\right)^{1 / 2}} \quad f_{R, n}^{i} \approx-f_{L, n}^{i} \quad S\left(L, m_{W, n}\right)=0
\end{aligned}
$$

From eq. (5.21), only the vector modes couple to the electroweak Goldstones. The sum rule now becomes

$$
\sum_{n} \frac{g_{W, n}^{2}}{m_{W, n}^{2}} \approx \frac{1}{3 f_{h}^{2}}
$$

Thus, at the scale of the first resonance effectively only one vector state appears in the Goldstone boson scattering amplitude. On the other hand, its coupling is stronger than in the Higgs limit by the factor $\sim \sqrt{2}$ :

$$
g_{W, 1}^{2} \approx \frac{m_{W, 1}^{2}}{3 f_{h}^{2}}
$$

\section{Gauge boson scattering amplitudes}

We come back to discussing the scattering of longitudinally polarized electroweak gauge bosons. In the following we discuss the specific case $W_{L} Z_{L} \rightarrow W_{L} Z_{L}$. The other scattering processes follow precisely the same logic. Quite generally, in the 5D models the scattering amplitude of the corresponding Goldstone fields has the form

$$
\mathcal{M}_{G^{+} G^{3} \rightarrow G^{+} G^{3}}=-\frac{g_{h}^{2}}{v^{2}} \frac{t m_{h}^{2}}{t-m_{h}^{2}}-\sum_{n} g_{C, n}^{2}\left(\frac{t-s}{u-m_{n}^{2}}+\frac{t-u}{s-m_{n}^{2}}\right)
$$

The sum runs over all charged vector boson states and $g_{C, n}$ denotes the couplings of the electroweak Goldstone bosons with the charged vector resonances. The first term comes from the self-interactions of the Goldstones and triple vertices with the physical Higgs boson, as in eq. (4.24). In the $5 \mathrm{D}$ models we consider, the quartic coupling is always correlated with that of the Higgs-Goldstone coupling and is given by $g_{h}^{2} / v^{2}$. 
From the Goldstone amplitude we can extract the dominant term in the scattering amplitude $W_{L} Z_{L} \rightarrow W_{L} Z_{L}$. At energies above the $m_{W}$ mass, but below the Higgs mass and the resonances scale, the amplitude contains the term that grows quadratically with energy:

$$
\mathcal{M}_{G^{+} G^{3} \rightarrow G^{+} G^{3}} \approx\left(\frac{g_{h}^{2}}{v^{2}}+3 \sum_{n>0} \frac{g_{C, n}^{2}}{m_{n}^{2}}\right) t \quad t<m_{h}
$$

The second term is the contribution of the heavy charged resonances (the light $W$ is omitted in this sum) that, at low energies, induce an effective four-Goldstone vertex. In fact, at this order the behaviour of the amplitude below the Higgs mass follows from the low energy theorems [16], $\mathcal{M} \approx t / \rho v^{2}$. In our $5 \mathrm{D}$ models, $\rho \approx 1+\mathcal{O}\left(v^{2} / M_{\mathrm{KK}}^{2}\right)$ due to the custodial symmetry. We thus conclude that the amplitude must grow like $t / v^{2}$. This tells us that the contribution of the resonances should adjust appropriately and there should be the sum rule

$$
3 \sum_{n>0} \frac{g_{C, n}^{2}}{m_{n}^{2}} \approx \frac{1-g_{h}^{2}}{v^{2}}
$$

There should also be an analogous sum rule involving neutral resonances. In several limiting cases, we have derived these precise sum rules through analytical calculations in 5D.

At energies above the Higgs mass, the first term in eq. (6.1) does not contribute to the quadratic growth. The effective contribution of the heavy resonances remains. The amplitude can be approximated:

$$
\mathcal{M}_{G^{+} G^{3} \rightarrow G^{+} G^{3}} \approx \frac{1-g_{h}^{2}}{v^{2}} t \quad m_{h}<E<m_{1}
$$

If $g_{h}<1$ the amplitude still grows quadratically, but slower (unless we are in the Higgsless limit where $g_{h}=0$ ). The quadratic growth is further softened around the first resonance mass. We can approximate

$$
\begin{aligned}
\mathcal{M}_{G^{+} G^{3} \rightarrow G^{+} G^{3}} & \approx\left(\frac{1-g_{h}^{2}}{v^{2}}-3 \frac{g_{C, 1}^{2}}{m_{C, 1}^{2}}\right) t-g_{C, 1}^{2}\left(\frac{t-s}{u-m_{C, 1}^{2}}+\frac{t-u}{s-m_{C, 1}^{2}}\right) \\
E & \sim m_{1}
\end{aligned}
$$

Above the first resonance the coefficient of the growing terms is diminished by $3 g_{1}^{2} / m_{1}^{2}$.

In the model of section 4 with the Higgs on the brane we found $g_{h}=(v / \tilde{v})^{3}$. This implies that $\left(1-g_{h}^{2}\right) / v^{2} \approx 3 / f_{h}^{2}$ in the Higgs limit, and $\left(1-g_{h}^{2}\right) / v^{2}=1 / v^{2} \approx 1 / f_{h}^{2}$ in the Higgsless limit. Thus the growth of the amplitude below the resonance scale is controlled by the scale $f_{h}$, although the coefficient varies depending on which limit we consider. In the model of section 5 with a pseudo-goldstone Higgs boson we found $g_{h} \approx \cos \left(\tilde{v} / f_{h}\right)$. This implies $\left(1-g_{h}^{2}\right) / v^{2} \approx 1 / f_{h}^{2}$ both in the Higgs limit and in the Higgsless limit.

In both models, unitarity is restored by resonances at the scale $M_{\mathrm{KK}}$. How efficiently the restoration proceeds, depends on the coupling strength of the lowest lying resonance(s), which is clearly model dependent. Nevertheless, in the 5D models we have studied we were able to find useful approximations for these couplings. The results for general backgrounds 
were given in section 4.4 and section 5.4. In order to get some feeling about validity of our estimates we now present the exact results for numerical calculations in $\mathrm{AdS}_{5}$.

We take the Planck/TeV hierarchy between UV and IR brane corresponding to $a_{L}^{-1}=$ $10^{15}$. The volume factor is then $\mathcal{V} \approx k L \approx 35$, the KK scale $M_{\mathrm{KK}} \approx \pi k a_{L}$, the decay constant $g_{L} f_{h} \approx 2 k a_{L} / \sqrt{\mathcal{V}}$.

In the model of section 4 the masses of lightest resonances are found as

$$
m_{W, 1} \approx 0.77 M_{\mathrm{KK}} \quad m_{W, 2} \approx 0.78 M_{\mathrm{KK}}
$$

in the Higgs limit and

$$
m_{W, 1} \approx 0.78 M_{\mathrm{KK}} \quad m_{W, 2} \approx 1.22 M_{\mathrm{KK}}
$$

in the Higgsless limit. In the Higgs limit we find the couplings $g_{W, 1} \approx 8.2 g_{W}, g_{W, 2} \approx 8.3 g_{W}$. Thus, at the resonance scale there are two almost degenerate vector states with comparable couplings to the electroweak Goldstone bosons. These two states saturate $66 \%$ of the sum rule (4.33). In the Higgsless limit the couplings are $g_{W, 1} \approx-8.1 g_{W}, g_{W, 2} \approx 0.1 g_{W}$, resulting in $96 \%$ of the sum rule (4.36) being saturated by the first resonance. Thus the estimate below eq. (4.36) perfectly captures the coupling of the first resonance. $W_{2}$ is the axial resonance and it approximately decouples from the electroweak Goldstone bosons. Avoiding violation of unitarity in the Higgsless limit requires $M_{\mathrm{KK}} \lesssim 1.5 \mathrm{TeV}$ (that is $\left.m_{W, 1} \lesssim 1.2 \mathrm{TeV}\right)$. Since $M_{\mathrm{KK}} \approx \pi \sqrt{\mathcal{V}} m_{W} \sim 1.5 \mathrm{TeV}$, the bound is saturated with the current choice of the hierarchy parameter $a_{L}^{-1} \sim 10^{15}$.

In the model of section 5 we find the masses of the lightest resonances

$$
m_{W, 1} \approx 0.8 M_{\mathrm{KK}} \quad m_{\bar{W}, 1} \approx 0.8 M_{\mathrm{KK}} \quad m_{W, 2} \approx 1.2 M_{\mathrm{KK}}
$$

where these masses are independent of the limit we consider.

In the Higgs limit we find $g_{W, 1} \approx-g_{\bar{W}, 1} \approx-5.7 g_{W}$ while $g_{W, 2} \approx 0$. In the Higgsless limit the coupling $g_{W, 1} \approx-8 g_{W}$, while $g_{\bar{W}, 1}=0$. In both cases, $95 \%$ of the respective sum rule is saturated by the first resonances.

The picture that emerges in both models is that the scattering amplitude is almost entirely unitarized at the scale of the first resonance. A distinctive feature of the Higgs limit this is that there are two almost degenerate resonances that contribute to unitarisation, while in the Higgsless limit just one resonance does most of the job.

\section{Conclusions}

New strong interactions as a cut-off to the SM are an interesting alternative to supersymmetry as an explanation of the origin of the electroweak scale. Before the LHC experiment tells us more about what nature has chosen, it is important to investigate the possible ways strong interactions could manifest themselves in the scattering of longitudinally polarised vector bosons. This is necessary for staying tuned in to the experimental analysis for various options. 
We have studied models of electroweak breaking formulated in 5D warped space. By relying on the heuristic link to strongly interacting theories in $4 \mathrm{D}$, or simply referring to 5D models of electroweak symmetry breaking, we have a technical, perturbative, means to investigate the detailed dynamics of the longitudinally polarised electroweak gauge bosons.

In this paper we have studied the dynamics of gauge bosons in two, previously proposed, 5D models of electroweak symmetry breaking. Using the powerful mass eigenstate technique in a general background [12, 13] we have calculated the mass spectrum of the vector resonances and their couplings, as well as the couplings of the physical 'composite' Higgs boson to the Goldstone bosons eaten by the $W$ and $Z$ gauge bosons. Using this input we calculated scattering amplitudes of the longitudinally polarised vector bosons using the equivalence theorem. This allowed us to discuss the role of various contributions in unitarising these scattering amplitudes.

The picture that emerges from the 5D models we studied is somewhat different from the expectations based on simple unitarisation procedures of the effective chiral Lagrangians $(\mathrm{EChL})$ [17]. In the EChL approach, unitarisation is generally achieved through both vector and scalar resonances. In the 5D models we consider, unitarisation is completed entirely by vector resonances, whose number and properties are quite constrained. Moreover, scalar resonances are not present in the spectrum at all, which is a consequence of the particular realization of electroweak symmetry breaking in our 5D models: by an IR brane field in the case of $\mathrm{SU}(2)_{L} \times \mathrm{SU}(2)_{R}$ or by the fifth component of the gauge field in the case of $\mathrm{SO}(5)$. In the holographic language, this can be interpreted as a limit in which the condensates that break symmetries of the strongly coupled sector are composite operators of infinite scaling dimension. Therefore the models we have considered here should be compared to the subset of EChL scenarios in which only vector resonances appear.

On the other hand, in 5D models in which the electroweak symmetry is broken, at least in part, by a vev of a bulk scalar field, the KK modes of that scalar do play a role in unitarization. However, as long as the 5D setup is not fine-tuned, the bulk Higgs vev must be sharply peaked towards IR, so that the effective description by symmetry breaking on the IR brane should be accurate and the role of the scalar resonances in these more general models is not expected to be prominent. Models with a bulk Higgs field are much more complicated at the technical level and we postpone their quantitative study for future publications.

Our explicit calculation in two very different models allows us to extract quite general features, hopefully common to any strongly interacting cut-off to the SM that incorporates vector resonances. These are, first of all, physical scales that emerge and characterise the gauge boson dynamics. We have identified four such scales: the electroweak scale $v$, the Higgs boson decay constant or equivalently the Higgs boson composite scale $f_{h}$, the resonance scale $M_{\mathrm{KK}}$ and finally the cut-off scale $\Lambda$ of the strongly interacting sector itself. Particularly interesting are the relative values of the scale $v$ versus $f_{h}$, and $f_{h}$ versus $M_{\mathrm{KK}}$ which fully determine the unitarity violation and restoration in the gauge bosons scattering amplitudes. We have found that resonances can appear over a broad range of energies, primarily determined by the geometry of the fifth dimension. However constraints from electroweak precision tests generally require these resonances to be heavy $(\gtrsim 3 \mathrm{TeV})$. 
Another interesting common feature of the two models is that they smoothly interpolate between the composite Higgs limit and the Higgsless limit, depending on the relative magnitude of the scales mentioned above. Thus we have explicit examples in which the strong dynamics is not as simple as is usually assumed in Higgsless models based on $\mathrm{SU}(2) \times \mathrm{SU}(2) \rightarrow \mathrm{SU}(2)$ and the gauge boson dynamics may lie between the composite Higgs and Higgsless limits. It is interesting by itself that in the Higgsless limit we have identified a new class of Higgsless models where the scalar particle does not play any role in the unitarisation of the scattering amplitudes but remains in the spectrum.

The question of whether the effects of the linear growth of the longitudinal scattering amplitude and its unitarity restoration can be detected at the LHC is vital. Providing a rigorous answer is certainly a hard task, given difficult QCD backgrounds. Unfortunately, the existing studies are not conclusive as they employ the language of chiral lagrangians [17] and they concentrate on the resonant effects. No studies of the signal and backgrounds have been done in a 5D framework where resonances are realized in a fully consistent way. Furthermore, since vector resonances in realistic models are expected to be quite heavy, $\gtrsim 3 \mathrm{TeV}$, because of electroweak precision tests, it would be important to investigate if the strong scattering effects can be also detected away from the resonant regions. Initial results [18] suggest that this may be possible and the results of a more complete analysis promise to be very interesting. To truly understand the experimental signature of the $5 \mathrm{D}$ models we consider would require a detailed Monte Carlo analysis. We leave this for a future work.

\section{Acknowledgments}

We thank Piotr Chankowski for his contributions to this projects at its early stage. SP would like to thank Gian Giudice for useful discussions. The work of AF and SP was supported by the European Community Contract MRTN-CT-2004-503369 for the years 2004-2008 and by the MEiN grant 1 P03B 09929 for the years 2005-2007. The work of JPR was funded under the FP6 Marie Curie contract MTKD-CT-2005-029466.

\section{A. Warped trigonometry}

The equation of motion

$$
\partial_{5}\left(a^{2}\left(x_{5}\right) \partial_{5} f\left(x_{5}\right)\right)+z^{2} f\left(x_{5}\right)=0
$$

has two independent solutions. Denote them $C\left(x_{5}, z\right)$ and $S\left(x_{5}, z\right)$. We choose them such their boundary conditions as

$$
C(0, z)=1 \quad C^{\prime}(0, z)=0 \quad S(0, z)=0 \quad S^{\prime}(0, z)=z
$$

so that in flat space they reduce to the familiar cosine and sine. The Wronskian relation

$$
S^{\prime}\left(x_{5}, z\right) C\left(x_{5}, z\right)-C^{\prime}\left(x_{5}, z\right) S\left(x_{5}, z\right)=z a^{-2}\left(x_{5}\right)
$$

is the warped analog of $\sin ^{2}+\cos ^{2}=1$. 
Integrating eq. (A.1) twice, we obtain the integral representation of the warped trigs

$$
\begin{aligned}
& C\left(x_{5}, z\right)=1-z^{2} \int_{0}^{x_{5}} a^{-2}(y) \int_{0}^{y} C\left(y^{\prime}, z\right) \\
& S\left(x_{5}, z\right)=z \int_{0}^{x_{5}} a^{-2}(y)-z^{2} \int_{0}^{x_{5}} a^{-2}(y) \int_{0}^{y} S\left(y^{\prime}, z\right)
\end{aligned}
$$

from which follows the expansion at small $z$ :

$$
\begin{aligned}
& C\left(x_{5}, z\right)=1-z^{2} \int_{0}^{x_{5}} y a^{-2}(y)+z^{4} \int_{0}^{x_{5}} a^{-2}(y) \int_{0}^{y} \int_{0}^{y^{\prime}} y^{\prime \prime} a^{-2}\left(y^{\prime \prime}\right)+\ldots \\
& S\left(x_{5}, z\right)=z \int_{0}^{x_{5}} a^{-2}(y)-z^{3} \int_{0}^{x_{5}} a^{-2}(y) \int_{0}^{y} \int_{0}^{y^{\prime}} a^{-2}\left(y^{\prime \prime}\right)+\ldots
\end{aligned}
$$

For $z$ and $y$ such that $z^{2} / a^{2} \gg 1$ we have another useful approximation.

$$
\begin{aligned}
& C\left(x_{5}, z\right) \approx a^{-1 / 2}\left(x_{5}\right) \alpha \cos \left(z \int_{L}^{x_{5}} a^{-1}(y)+\phi_{\alpha}\right) \\
& S\left(x_{5}, z\right) \approx-a^{-1 / 2}\left(x_{5}\right) \beta \cos \left(z \int_{L}^{x_{5}} a^{-1}(y)+\phi_{\beta}\right)
\end{aligned}
$$

where the four real parameters $\alpha, \beta, \phi_{\alpha}, \phi_{\beta}$ are bound to satisfy $\beta \alpha \sin \left(\phi_{\beta}-\phi_{\alpha}\right)=$ 1. Moreover, for metrics highly warped toward the IR brane we have an approximate relation $C(L, z) \approx-z L S(L, z), C^{\prime}(L, z) \approx-z L S^{\prime}(L, z)$ that follows from the perturbation expansion (A.5), with $y \rightarrow L$ in the integrals.

Let us see the warped sines and cosines in some particular, solvable backgrounds.

For flat space, $a\left(x_{5}\right)=1$, we get the familiar trigonometric functions:

$$
C\left(x_{5}, z\right)=\cos \left(z x_{5}\right) \quad S\left(x_{5}, z\right)=\sin \left(z x_{5}\right)
$$

For $\mathrm{AdS}_{5}$ we insert $a\left(x_{5}\right)=e^{-k x_{5}}$ and almost as easily solve eq. (A.1) in terms of Bessel functions. The solution is $a^{-1} Z_{1}(m / k a)$ (note also that $\left[a^{-1} Z_{1}(m / k a)\right]^{\prime}=z a^{-2} Z_{0}(m / k a)$ ) and we pick up the following combinations

$$
\begin{aligned}
& C\left(x_{5}, z\right)=\frac{\pi z}{2 k} a^{-1}\left(x_{5}\right)\left[Y_{0}\left(\frac{z}{k}\right) J_{1}\left(\frac{z}{k a\left(x_{5}\right)}\right)-J_{0}\left(\frac{z}{k}\right) Y_{1}\left(\frac{z}{k a\left(x_{5}\right)}\right)\right] \\
& S\left(x_{5}, z\right)=\frac{\pi z}{2 k} a^{-1}\left(x_{5}\right)\left[-Y_{1}\left(\frac{z}{k}\right) J_{1}\left(\frac{z}{k a\left(x_{5}\right)}\right)+J_{1}\left(\frac{z}{k}\right) Y_{1}\left(\frac{z}{k a\left(x_{5}\right)}\right)\right]
\end{aligned}
$$

Another solvable background is that with a power law warp factor $a\left(x_{5}\right)=\left(1-\frac{k x_{5}}{\gamma-1}\right)^{\gamma}$ For $\gamma \rightarrow \infty$ we recover the exponential warp factor of the RS model. The solutions to eq. (A.1) can be written, similarly as in the $\mathrm{AdS}_{5}$ case, in terms of the Bessel functions,

$$
\begin{aligned}
& C\left(x_{5}, z\right)=\frac{\pi z}{2 k} a^{-1+\frac{1}{2 \gamma}}\left[Y_{\frac{1}{2 \gamma-2}}\left(\frac{z}{k}\right) J_{\frac{2 \gamma-1}{2 \gamma-2}}\left(\frac{z}{k a^{1-\frac{1}{\gamma}}}\right)-J_{\frac{1}{2 \gamma-2}}\left(\frac{z}{k}\right) Y_{\frac{2 \gamma-1}{2 \gamma-2}}\left(\frac{z}{k a^{1-\frac{1}{\gamma}}}\right)\right] \\
& S\left(x_{5}, z\right)=\frac{\pi z}{2 k} a^{-1+\frac{1}{2 \gamma}}\left[-Y_{\frac{2 \gamma-1}{2 \gamma-2}}\left(\frac{z}{k}\right) J_{\frac{2 \gamma-1}{2 \gamma-2}}\left(\frac{z}{k a^{1-\frac{1}{\gamma}}}\right)+J_{\frac{2 \gamma-1}{2 \gamma-2}}\left(\frac{z}{k}\right) Y_{\frac{2 \gamma-1}{2 \gamma-2}}\left(\frac{z}{k a^{1-\frac{1}{\gamma}}}\right)\right]
\end{aligned}
$$




\section{B. $\mathrm{SU}(3)_{c} \times \mathrm{SU}(2)_{L} \times \mathrm{SU}(2)_{R} \times \mathrm{U}(1)_{X}$ : profiles and couplings}

We collect here various technical details concerning the model of section 3 . We list the profiles that follow from solving the boundary conditions on the IR brane. For the $\mathrm{W}$ tower

$$
\begin{aligned}
f_{L, n}^{i} & =\alpha_{W, n} C\left(x_{5}, m_{W, n}\right) \\
f_{R, n}^{i} & =-\frac{g_{R}}{g_{L}} \alpha_{W, n} \frac{C^{\prime}\left(L, m_{W, n}\right)}{S^{\prime}\left(L, m_{W, n}\right)} S\left(x_{5}, m_{W, n}\right) \\
\left(\alpha_{W, n}\right)^{-2} & =\int_{0}^{L} d y\left\{C^{2}\left(y, m_{W, n}\right)+\frac{g_{R}^{2}}{g_{L}^{2}}\left(\frac{C^{\prime}\left(L, m_{W, n}\right)}{S^{\prime}\left(L, m_{W, n}\right)}\right)^{2} S^{2}\left(y, m_{W, n}\right)\right\}
\end{aligned}
$$

For the photon tower

$$
\begin{aligned}
f_{L, n}^{3} & =\sin \theta_{W} \alpha_{\gamma, n} C\left(x_{5}, m_{n}\right) \\
f_{R, n}^{3} & =s_{x} \cos \theta_{W} \alpha_{\gamma, n} C\left(x_{5}, m_{n}\right) \\
f_{X, n} & =c_{x} \cos \theta_{W} \alpha_{\gamma, n} C\left(x_{5}, m_{n}\right) \\
\left(\alpha_{\gamma, n}\right)^{-2} & =\int_{0}^{L} d y C^{2}\left(y, m_{n}\right)
\end{aligned}
$$

For the $\mathrm{Z}$ tower

$$
\begin{aligned}
f_{L, n}^{3} & =\cos \theta_{W} \alpha_{Z, n} C\left(x_{5}, m_{Z, n}\right) \\
f_{R, n}^{3} & =-s_{x} \sin \theta_{W} \alpha_{Z, n} C\left(x_{5}, m_{Z, n}\right)-\frac{c_{x}^{2}}{s_{x}} \sin \theta_{W} \alpha_{Z, n} \frac{C^{\prime}\left(L, m_{Z, n}\right)}{S^{\prime}\left(L, m_{Z, n}\right)} S\left(x_{5}, m_{Z, n}\right) \\
f_{X, n} & =-c_{x} \sin \theta_{W} \alpha_{Z, n} C\left(x_{5}, m_{n}\right)+c_{x} \sin \theta_{W} \alpha_{Z, n} \frac{C^{\prime}\left(L, m_{Z, n}\right)}{S^{\prime}\left(L, m_{Z, n}\right)} S\left(x_{5}, m_{Z, n}\right) \\
\left(\alpha_{Z, n}\right)^{-2} & =\int_{0}^{L} d y\left\{C^{2}\left(x_{5}, m_{Z, n}\right)+\frac{c_{x}^{2}}{s_{x}^{2}} \sin ^{2} \theta_{W}\left(\frac{C^{\prime}\left(L, m_{Z, n}\right)}{S^{\prime}\left(L, m_{Z, n}\right)}\right)^{2} S^{2}\left(x_{5}, m_{Z, n}\right)\right\}
\end{aligned}
$$

The quantization conditions are given in eqs. (4.13), (4.14), 4.15). Expanding $C$ and $S$ at small $m$ allows to estimate the masses of $\mathrm{W}$ and $\mathrm{Z}$. Including corrections of order $v^{2} / M_{\mathrm{KK}}^{2}$ we find

$$
\begin{aligned}
m_{W}^{2} \approx g_{L}^{2} \frac{\tilde{v}^{2}}{4}\left(1+\frac{\tilde{v}^{2}}{4}\left[g_{L}^{2} L^{-1} \int_{0}^{L} \int_{0}^{y} y^{\prime} a^{-2}\left(y^{\prime}\right)-g_{L}^{2} \int_{0}^{L} y a^{-2}(y)-g_{R}^{2} L \int_{0}^{L} a^{-2}(y)\right]\right) \\
m_{Z}^{2} \approx\left(g_{L}^{2}+g_{Y}^{2}\right) \frac{\tilde{v}^{2}}{4}\left(1+\frac{\tilde{v}^{2}}{4}\left[\left(g_{L}^{2}+g_{Y}^{2}\right) L^{-1} \int_{0}^{L} \int_{0}^{y} y^{\prime} a^{-2}\left(y^{\prime}\right)\right.\right. \\
\left.\left.\quad-g_{L}^{2} \int_{0}^{L} y a^{-2}(y)-g_{R}^{2} L \int_{0}^{L} a^{-2}(y)+g_{Y}^{2} \int_{0}^{L} \int_{0}^{y} a^{-2}\left(y^{\prime}\right)\right]\right)
\end{aligned}
$$

From dimensional analysis one would expect the integrals in the above expression to be of order $1 / M_{\mathrm{KK}}^{2}$. However, some of the integrals scale linearly with $L$, therefore they can be enhanced when the volume factor is large. In such a case, the corrections turn out to be $\mathcal{O}\left(v^{2} / f_{h}^{2}\right)$ rather than $\mathcal{O}\left(v^{2} / M_{\mathrm{KK}}^{2}\right)$. 
The Goldstone profiles corresponding to $\mathrm{W}$ and $\mathrm{Z}$ are given by

$$
\begin{aligned}
\bar{f}_{L, W}^{i} & =\alpha_{W} m_{W}^{-1} C^{\prime}\left(x_{5}, m_{W}\right) \\
\bar{f}_{R, W}^{i} & =-\frac{g_{R}}{g_{L}} \alpha_{W} \frac{C^{\prime}\left(L, m_{W}\right)}{S^{\prime}\left(L, m_{W}\right)} m_{W}^{-1} S^{\prime}\left(x_{5}, m_{W}\right) \\
\tilde{f}_{W}^{i} & =-\alpha_{W} \frac{2}{m_{W} \sqrt{L} g_{L} \tilde{v}} a_{L}^{2} C^{\prime}\left(L, m_{W}\right) \\
\bar{f}_{L, Z}^{3} & =\cos \theta_{W} \alpha_{Z} m_{Z}^{-1} C^{\prime}\left(x_{5}, m_{Z}\right) \\
\bar{f}_{R, Z}^{3} & =-s_{x} \sin \theta_{W} \alpha_{Z} m_{Z}^{-1} C^{\prime}\left(x_{5}, m_{Z}\right)-\frac{c_{x}^{2}}{s_{x}} \sin \theta_{W} \alpha_{Z} \frac{C^{\prime}\left(L, m_{Z}\right)}{S^{\prime}\left(L, m_{Z}\right)} m_{Z}^{-1} S^{\prime}\left(x_{5}, m_{Z}\right) \\
\bar{f}_{X, Z} & =-c_{x} \sin \theta_{W} \alpha_{Z} m_{Z}^{-1} C^{\prime}\left(x_{5}, m_{Z}\right)+c_{x} \sin \theta_{W} \alpha_{Z} \frac{C^{\prime}\left(L, m_{Z}\right)}{S^{\prime}\left(L, m_{Z}\right)} m_{Z}^{-1} S^{\prime}\left(x_{5}, m_{Z}\right) \\
\tilde{f}_{Z}^{3} & =-\alpha_{Z} \frac{2}{m_{Z} \sqrt{L} \sqrt{g_{L}^{2}+g_{Y}^{2}} \tilde{v}} a_{L}^{2} C^{\prime}\left(L, m_{Z}\right)
\end{aligned}
$$

The expansion of these profiles for small masses can be done with the help of eq. (A.5). To lowest order in $m_{W}, m_{Z}$, we derive eq. (4.21).

The profiles serve to establish the couplings of the Goldstones to the resonances. Working out the relevant terms in the 5D action the couplings to the neutral gauge bosons are given by

$$
\begin{aligned}
g_{N, n}= & \frac{m_{W}^{2}}{\sqrt{L}} \int_{0}^{L} d y a^{-2}(y)\left\{g_{L} y^{2} f_{L, n}^{3}(y)+\frac{g_{R}^{3}}{g_{L}^{2}} L^{2} f_{R, n}^{3}(y)\right\} \\
& +\frac{1}{2} \sqrt{L} \frac{v^{2}}{\tilde{v}^{2}}\left(g_{L} f_{L, n}^{3}(L)+g_{R} f_{R, n}^{3}(L)\right)
\end{aligned}
$$

while those to the charged gauge bosons

$$
\begin{aligned}
g_{C, n}= & \frac{m_{W}^{2}}{\sqrt{L}} \int_{0}^{L} d y a^{-2}(y)\left\{g_{L} y^{2} f_{L, n}^{i}(y)+\frac{g_{R}^{3}}{g_{L}^{2}} L\left(s_{x}^{2} y+c_{x}^{2} L\right) f_{R, n}^{i}(y)\right\} \\
& +\frac{1}{2} \sqrt{L} \frac{v^{2}}{\tilde{v}^{2}}\left(g_{L} f_{L, n}^{i}(L)+g_{R} f_{R, n}^{i}(L)\right)
\end{aligned}
$$

Due to the $a^{-2}$ factor, the integrals are dominated by the behaviour of the profiles near the IR brane. Therefore it is sane to replace $y \rightarrow L$ under the integrals. Moreover, we set $g_{L}=g_{R}$ for simplicity. This yields

$$
\begin{aligned}
g_{W, n} \approx g_{L} \sqrt{L} \int_{0}^{L} a^{-2}(y)\left\{\frac{1}{2} \frac{v^{2}}{\tilde{v}^{2}} \delta(L)+m_{W}^{2} L a^{-2}(y)\right\} \\
\frac{C\left(y, m_{W, n}\right)-\frac{C^{\prime}\left(L, m_{W, n}\right)}{S^{\prime}\left(L, m_{W, n}\right)} S\left(y, m_{W, n}\right)}{\left[\int_{0}^{L} d y\left\{C^{2}\left(y, m_{n}\right)+\left(\frac{C^{\prime}\left(L, m_{n}\right)}{S^{\prime}\left(L, m_{n}\right)}\right)^{2} S^{2}\left(y, m_{n}\right)\right\}\right]^{1 / 2}}
\end{aligned}
$$




$$
\begin{gathered}
g_{Z, n} \approx \frac{g_{L}^{2}-g_{Y}^{2}}{\sqrt{g_{L}^{2}+g_{Y}^{2}}} \sqrt{L} \int_{0}^{L} a^{-2}(y)\left\{\frac{1}{2} \frac{v^{2}}{\tilde{v}^{2}} \delta(L)+m_{W}^{2} L a^{-2}(y)\right\} \\
\frac{C\left(y, m_{Z, n}\right)-\frac{C^{\prime}\left(L, m_{Z, n}\right)}{S^{\prime}\left(L, m_{Z, n}\right)} S\left(y, m_{Z, n}\right)}{\left[\int_{0}^{L} d y\left\{C^{2}\left(y, m_{n}\right)+\cos 2 \theta_{W}\left(\frac{C^{\prime}\left(L, m_{n}\right)}{S^{\prime}\left(L, m_{n}\right)}\right)^{2} S^{2}\left(y, m_{n}\right)\right\}\right]^{1 / 2}} \\
g_{\gamma, n} \approx 2 e \sqrt{L} \int_{0}^{L} a^{-2}(y)\left\{\frac{1}{2} \frac{v^{2}}{\tilde{v}^{2}} \delta(L)+m_{W}^{2} L a^{-2}(y)\right\} \frac{C\left(y, m_{\gamma, n}\right)}{\left[\int_{0}^{L} C^{2}\left(y, m_{\gamma, n}\right)\right]^{1 / 2}}
\end{gathered}
$$

\section{C. $\mathrm{SU}(3)_{c} \times \mathrm{SO}(5) \times \mathrm{U}(1)_{X}$ : profiles and couplings}

We move to the holographic model of a pseudo-Goldstone Higgs boson. Choosing the direction of the Higgs vev as $\left\langle A_{5}^{\hat{4}}\right\rangle=\tilde{v} a^{-2} / \sqrt{\int_{0}^{L} a^{-2}(y)}$, the Wilson-line matrix is given by

$$
\omega\left(x_{5}, \tilde{v}\right)=\left[\begin{array}{ccccc}
1 & 0 & 0 & 0 & 0 \\
0 & 1 & 0 & 0 & 0 \\
0 & 0 & 1 & 0 & 0 \\
0 & 0 & 0 & \cos \left(\frac{\tilde{v}}{f\left(x_{5}\right)}\right) & -\sin \left(\frac{\tilde{v}}{f\left(x_{5}\right)}\right) \\
0 & 0 & 0 & \sin \left(\frac{v}{f\left(x_{5}\right)}\right) & \cos \left(\frac{\tilde{v}}{f\left(x_{5}\right)}\right)
\end{array}\right] \quad f\left(x_{5}\right)=\frac{\sqrt{2} \sqrt{\int_{0}^{L} a^{-2}(y)}}{g \sqrt{L} \int_{0}^{x_{5}} a^{-2}(y)}
$$

This yields the link between the profiles with zero and non-zero vev:

$$
\begin{aligned}
f_{L}^{a}\left(x_{5}, \tilde{v}\right) & =\frac{1+\cos (\tilde{v} / f)}{2} f_{L}^{a}\left(x_{5}\right)+\frac{1-\cos (\tilde{v} / f)}{2} f_{R}^{a}\left(x_{5}\right)+\frac{\sin (\tilde{v} / f)}{\sqrt{2}} f_{C}^{a}\left(x_{5}\right) \\
f_{R}^{a}\left(x_{5}, \tilde{v}\right) & =\frac{1-\cos (\tilde{v} / f)}{2} f_{L}^{a}\left(x_{5}\right)+\frac{1+\cos (\tilde{v} / f)}{2} f_{R}^{a}\left(x_{5}\right)-\frac{\sin (\tilde{v} / f)}{\sqrt{2}} f_{C}^{a}\left(x_{5}\right) \\
f_{C}^{a}\left(x_{5}, \tilde{v}\right) & =-\frac{\sin (\tilde{v} / f)}{\sqrt{2}} f_{L}^{a}\left(x_{5}\right)+\frac{\sin (\tilde{v} / f)}{\sqrt{2}} f_{R}^{a}\left(x_{5}\right)+\cos (\tilde{v} / f) f_{C}^{a}\left(x_{5}\right) \\
f_{C}^{4}\left(x_{5}, \tilde{v}\right) & =f_{C}^{4}\left(x_{5}\right) \\
f_{X}(\tilde{v}) & =f_{X}\left(x_{5}\right)
\end{aligned}
$$

Inserting this into the IR boundary conditions we can find the mass eigenstates.

Charged. The charged gauge bosons are combinations of $L_{\mu}^{i}, R_{\mu}^{i}$ and $C_{\mu}^{i}$. In the charged sector we have two towers. In the one referred to as the $\bar{W}$ tower the masses do not depend on $\tilde{v}$. The quantization condition is simple:

$$
S^{\prime}\left(L, m_{\bar{W}, n}\right)=0
$$

This tower has the profiles:

$$
\begin{aligned}
f_{R, n}^{i} & =\alpha_{\bar{W}, n} \sqrt{2} \cos \left(\tilde{v} / f_{h}\right) S\left(x_{5}, m_{\bar{W}, n}\right) \\
f_{C, n}^{i} & =-\alpha_{\bar{W}, n} \sin \left(\tilde{v} / f_{h}\right) S\left(x_{5}, m_{\bar{W}, n}\right) \\
\left(\alpha_{\bar{W}, n}\right)^{-2} & =\left(1+\cos ^{2}\left(\tilde{v} / f_{h}\right)\right)^{2} \int_{0}^{L}\left[S\left(y, m_{\bar{W}, n}\right)\right]^{2}
\end{aligned}
$$


In the other tower, referred to as the $W$ tower, the masses do depend on $\tilde{v}$. The quantization condition is

$$
C^{\prime}\left(L, m_{W, n}\right) S\left(L, m_{W, n}\right)+\frac{1}{2} m_{W, n} a_{L}^{-2} \sin ^{2}\left(\frac{\tilde{v}}{f_{h}}\right)=0
$$

The corresponding profiles are

$$
\begin{aligned}
f_{L, n}^{i} & =\alpha_{W, n} C\left(x_{5}, m_{W, n}\right) \\
f_{R, n}^{i} & =-\alpha_{W, n} \frac{C^{\prime}\left(L, m_{W, n}\right)}{S^{\prime}\left(L, m_{W, n}\right)} S\left(x_{5}, m_{W, n}\right) \\
f_{C, n}^{i} & =-\alpha_{W, n} \frac{\sqrt{2} \cos \left(\tilde{v} / f_{h}\right)}{\sin \left(\tilde{v} / f_{h}\right)} \frac{C^{\prime}\left(L, m_{W, n}\right)}{S^{\prime}\left(L, m_{W, n}\right)} S\left(x_{5}, m_{W, n}\right) \\
\left(\alpha_{W, n}\right)^{-2} & =\int_{0}^{L}\left\{\left[C\left(y, m_{W, n}\right)\right]^{2}-\frac{C^{\prime}\left(L, m_{W, n}\right) C\left(L, m_{W, n}\right)}{S^{\prime}\left(L, m_{W, n}\right) S\left(L, m_{W, n}\right)}\left[S\left(y, m_{W, n}\right)\right]^{2}\right\}
\end{aligned}
$$

There is a light solution of the quantization condition proportional to $f_{h} \sin \left(\tilde{v} / f_{h}\right)$. This is the $W$ boson.

Neutral. The neutral gauge bosons are combinations of $L_{\mu}^{3}, R_{\mu}^{3}$ and $C_{\mu}^{3,4}$ and $X_{\mu}$. Three of them have $\tilde{v}$-independent masses. One is along the same group space direction as the Higgs vev, hence we refer to it as the Higgs tower. The quantization condition:

$$
S\left(L, m_{H, n}\right)=0
$$

The profile

$$
f_{C, n}^{4}=\alpha_{H, n} S\left(x_{5}, m_{H, n}\right) \quad\left(\alpha_{H, n}\right)^{-2}=\int_{0}^{L}\left[S\left(y, m_{H, n}\right)\right]^{2}
$$

There is no light (mode) in this tower.

Another is called the photon tower. The quantization condition:

$$
C^{\prime}\left(L, m_{\gamma, n}\right)=0
$$

The profiles

$$
\begin{aligned}
f_{L, n}^{3} & =\sin \theta_{W} \alpha_{\gamma, n} C\left(x_{5}, m_{\gamma, n}\right) \\
f_{R, n}^{3} & =s_{x} \cos \theta_{W} \alpha_{\gamma, n} C\left(x_{5}, m_{\gamma, n}\right) \\
f_{X, n} & =c_{x} \cos \theta_{W} \alpha_{\gamma, n} C\left(x_{5}, m_{\gamma, n}\right) \\
\left(\alpha_{\gamma, n}\right)^{-2} & =\int_{0}^{L}\left[C\left(y, m_{\gamma, n}\right)\right]^{2}
\end{aligned}
$$

The photon tower includes a massless eigenvector: the photon.

There is the $\bar{Z}$ tower, which is similar to the $\bar{W}$ tower and has no light mode. The quantization:

$$
S^{\prime}\left(L, m_{\bar{Z}, n}\right)=0
$$


The profiles

$$
\begin{aligned}
f_{R, n}^{3} & =-c_{x} \alpha_{\bar{Z}, n} \sqrt{2} \cos \left(\tilde{v} / f_{h}\right) S\left(x_{5}, m_{\bar{Z}, n}\right) \\
f_{X, n} & =s_{x} \alpha_{\bar{Z}, n} \sqrt{2} \cos \left(\tilde{v} / f_{h}\right) S\left(x_{5}, m_{\bar{Z}, n}\right) \\
f_{C, n}^{3} & =c_{x} \alpha_{\bar{Z}, n} \sin \left(\tilde{v} / f_{h}\right) S\left(x_{5}, m_{\bar{Z}, n}\right) \\
\left(\alpha_{\bar{Z}, n}\right)^{-2} & =\left(1+\cos ^{2}\left(\tilde{v} / f_{h}\right)-s_{x}^{2} \sin ^{2}\left(\tilde{v} / f_{h}\right)\right)^{2} \int_{0}^{L}\left[S\left(y, m_{\bar{Z}, n}\right)\right]^{2}
\end{aligned}
$$

Finally there is the $Z$ tower, where masses are sensitive to $\tilde{v}$. The quantization condition is very similar to that of the $\mathrm{W}$-tower,

$$
\cos ^{2} \theta_{W} C^{\prime}\left(L, m_{Z, n}\right) S\left(L, m_{Z, n}\right)+\frac{1}{2} m_{Z, n} a_{L}^{-2} \sin ^{2}\left(\frac{\tilde{v}}{f_{h}}\right)=0
$$

the difference being the cosine of the Weinberg angle. The profiles

$$
\begin{aligned}
f_{L, n}^{3} & =\cos \theta_{W} \alpha_{Z, n} C\left(x_{5}, m_{Z, n}\right) \\
f_{R, n}^{3} & =-s_{x} \sin \theta_{W} \alpha_{Z, n} C\left(x_{5}, m_{Z, n}\right)-c_{x}^{2} \cos \theta_{W} \alpha_{Z, n} \frac{C^{\prime}\left(L, m_{Z, n}\right)}{S^{\prime}\left(L, m_{Z, n}\right)} S\left(x_{5}, m_{Z, n}\right) \\
f_{X, n} & =-c_{x} \sin \theta_{W} \alpha_{Z, n} C\left(x_{5}, m_{Z, n}\right)+c_{x} \sin \theta_{W} \alpha_{Z, n} \frac{C^{\prime}\left(L, m_{Z, n}\right)}{S^{\prime}\left(L, m_{Z, n}\right)} S\left(x_{5}, m_{Z, n}\right) \\
f_{C, n}^{3} & =-\cos \theta_{W} \alpha_{Z, n} \frac{\sqrt{2} \cos \left(\tilde{v} / f_{h}\right)}{\sin \left(\tilde{v} / f_{h}\right)} \frac{C^{\prime}\left(L, m_{Z, n}\right)}{S^{\prime}\left(L, m_{Z, n}\right)} S\left(x_{5}, m_{Z, n}\right) \\
\left(\alpha_{Z, n}\right)^{-2} & =\int_{0}^{L}\left\{\left[C\left(y, m_{Z, n}\right)\right]^{2}-\frac{C^{\prime}\left(L, m_{Z, n}\right) C\left(L, m_{Z, n}\right)}{S^{\prime}\left(L, m_{Z, n}\right) S\left(L, m_{Z, n}\right)}\left[S\left(y, m_{Z, n}\right)\right]^{2}\right\}
\end{aligned}
$$

The $Z$ boson is the lightest solution of the quantization condition with the mass proportional to $f_{h} \sin \left(\tilde{v} / f_{h}\right)$.

We move to discussing the profiles of the Goldstones corresponding to the electroweak gauge bosons. The Goldstone profiles at zero vev are simply related to the corresponding gauge profiles, see eq. (5.10). The Goldstones eaten by $W$ and $Z$ have the following profile

$$
\begin{aligned}
& \bar{f}_{L, W}^{i}=\alpha_{W} m_{W}^{-1} C^{\prime}\left(x_{5}, m_{W}\right) \\
& \bar{f}_{R, W}^{i}=-\alpha_{W} \frac{C^{\prime}\left(L, m_{W}\right)}{S^{\prime}\left(L, m_{W}\right)} m_{W}^{-1} S^{\prime}\left(x_{5}, m_{W}\right) \\
& \bar{f}_{C, W}^{i}=-\alpha_{W} \frac{\sqrt{2} \cos \left(\tilde{v} / f_{h}\right)}{\sin \left(\tilde{v} / f_{h}\right)} \frac{C^{\prime}\left(L, m_{W}\right)}{S^{\prime}\left(L, m_{W}\right)} m_{W}^{-1} S^{\prime}\left(x_{5}, m_{W}\right) \\
& \bar{f}_{L, Z}^{3}=\cos \theta_{W} \alpha_{Z} m_{Z}^{-1} C^{\prime}\left(x_{5}, m_{Z}\right) \\
& \bar{f}_{R, Z}^{3}=-s_{x} \sin \theta_{W} \alpha_{Z} m_{Z}^{-1} C^{\prime}\left(x_{5}, m_{Z}\right)-c_{x}^{2} \cos \theta_{W} \alpha_{Z, n} \frac{C^{\prime}\left(L, m_{Z}\right)}{S^{\prime}\left(L, m_{Z}\right)} m_{Z}^{-1} S^{\prime}\left(x_{5}, m_{Z}\right) \\
& \bar{f}_{X, Z}=-c_{x} \sin \theta_{W} \alpha_{Z} m_{Z}^{-1} C^{\prime}\left(x_{5}, m_{Z, n}\right)+c_{x} \sin \theta_{W} \alpha_{Z} \frac{C^{\prime}\left(L, m_{Z}\right)}{S^{\prime}\left(L, m_{Z}\right)} m_{Z}^{-1} S^{\prime}\left(x_{5}, m_{Z}\right) \\
& \bar{f}_{C, Z}^{3}=-\cos \theta_{W} \alpha_{Z} \frac{\sqrt{2} \cos \left(\tilde{v} / f_{h}\right)}{\sin \left(\tilde{v} / f_{h}\right)} \frac{C^{\prime}\left(L, m_{Z}\right)}{S^{\prime}\left(L, m_{Z}\right)} S^{\prime}\left(x_{5}, m_{Z}\right)
\end{aligned}
$$


Expanding the warped trigs for a small $m$ yields the approximate expressions (5.19).

These approximate profiles allow us to determine the resonance couplings. The general formula for the charged ones is

$$
\begin{array}{r}
g_{C, n}=g \sqrt{L} \int_{0}^{L} a^{2}\left\{f_{L, n}^{i} \bar{f}_{L, Z}^{3} \bar{f}_{L, W}^{i}+f_{R, n}^{i} \bar{f}_{R, Z}^{3} \bar{f}_{R, W}^{i}+\frac{1}{2}\left(f_{L, n}^{i}+f_{R, n}^{i}\right) \bar{f}_{C, Z}^{3} \bar{f}_{C, W}^{i}\right. \\
\left.+\frac{1}{2} f_{C, n}^{i}\left(\bar{f}_{L, Z}^{3}+\bar{f}_{R, Z}^{3}\right) \bar{f}_{C, W}^{i}-\frac{1}{2} f_{C, n}^{i}\left(\bar{f}_{L, W}^{i}+\bar{f}_{R, W}^{i}\right) \bar{f}_{C, Z}^{3}\right\}
\end{array}
$$

(no summing over $i$ here). For the neutral ones,

$$
\begin{aligned}
g_{N, n}=g \sqrt{L} \int_{0}^{L} a^{2}\left\{f_{L, n}^{3} \bar{f}_{L, W}^{i} \bar{f}_{L, W}^{i}+f_{R, n}^{3} \bar{f}_{R, W}^{i} \bar{f}_{R, W}^{i}\right. & \\
& \left.+\frac{1}{2}\left(f_{L, n}^{3}+f_{R, n}^{3}\right) \bar{f}_{C, W}^{i} \bar{f}_{C, W}^{i} f_{C, n}^{3}\left(\bar{f}_{L, W}^{i}+\bar{f}_{R, W}^{i}\right) \bar{f}_{C, W}^{i}\right\}
\end{aligned}
$$

Inserting the approximate Goldstone profiles and the exact profiles of vector resonances we obtain the couplings

$$
\begin{aligned}
& g_{W, n} \approx \frac{g_{L}}{2} \frac{\sqrt{L} \int_{0}^{L} a^{-2}(y)\left\{C\left(y, m_{W, n}\right)-\frac{C^{\prime}\left(L, m_{W, n}\right)}{S^{\prime}\left(L, m_{W, n}\right)} S\left(y, m_{W, n}\right)\left(1-s_{x}^{2}(1-y / L)\right)\right\}}{\left[\int_{0}^{L} a^{-2}(y)\right]\left[\int_{0}^{L} C^{2}\left(y, m_{W, n}\right)-\frac{C^{\prime}\left(L, m_{W, n}\right) C\left(L, m_{W, n}\right)}{S^{\prime}\left(L, m_{W, n}\right) S\left(L, m_{W, n}\right)} S^{2}\left(y, m_{W, n}\right)\right]^{1 / 2}} \\
& g_{\hat{W}, n} \approx \frac{g_{L}}{\sqrt{2}} \frac{\cos \left(\tilde{v} / f_{h}\right)}{\sqrt{1+\cos ^{2}\left(\tilde{v} / f_{h}\right)}} \frac{\sqrt{L} \int_{0}^{L} a^{-2}(y) S\left(y, m_{\hat{W}, n}\right)}{\left[\int_{0}^{L} a^{-2}(y)\right]\left[\int_{0}^{L} S^{2}\left(y, m_{\hat{W}, n}\right)\right]^{1 / 2}} \\
& g_{Z, n} \approx \frac{g_{L}^{2}-g_{Y}^{2}}{2 \sqrt{g_{L}^{2}+g_{Y}^{2}}} \times \\
& \times \frac{\sqrt{L} \int_{0}^{L} a^{-2}(y)\left\{C\left(y, m_{Z, n}\right)-\frac{C^{\prime}\left(L, m_{Z, n}\right)}{S^{\prime}\left(L, m_{Z, n}\right)} S\left(y, m_{Z, n}\right)\left(1-\frac{2 g_{L}^{2} \cos ^{2}\left(\tilde{v} / f_{h}\right)}{g_{L}^{2}-g_{Y}^{2}}(1-y / L)\right)\right\}}{\left[\int_{0}^{L} a^{-2}(y)\right]\left[\int_{0}^{L} C^{2}\left(y, m_{Z, n}\right)-\frac{C^{\prime}\left(L, m_{Z, n}\right) C\left(L, m_{Z, n}\right)}{S^{\prime}\left(L, m_{Z, n}\right) S\left(L, m_{Z, n}\right)} S^{2}\left(y, m_{Z, n}\right)\right]^{1 / 2}} \\
& g_{\bar{Z}, n} \approx \frac{g_{L}}{\sqrt{2}} c_{x} \frac{\cos \left(\tilde{v} / f_{h}\right)}{\sqrt{1+\cos ^{2}\left(\tilde{v} / f_{h}\right)-s_{x}^{2} \sin ^{2}\left(\tilde{v} / f_{h}\right)}} \frac{\sqrt{L} \int_{0}^{L} a^{-2}(y) S\left(y, m_{\hat{Z}, n}\right)}{\left[\int_{0}^{L} a^{-2}(y)\right]\left[\int_{0}^{L} S^{2}\left(y, m_{\hat{Z}, n}\right)\right]^{1 / 2}} \\
& g_{\gamma, n} \approx e \frac{\sqrt{L} \int_{0}^{L} a^{-2}(y) C\left(y, m_{\gamma, n}\right)}{\left[\int_{0}^{L} a^{-2}(y)\right]\left[\int_{0}^{L} C^{2}\left(y, m_{\gamma, n}\right)\right]^{1 / 2}}
\end{aligned}
$$

The Higgs tower does not couple to the electroweak Goldstones.

\section{References}

[1] N. Arkani-Hamed, M. Porrati and L. Randall, Holography and phenomenology, JHEP 08 (2001) 017 hep-th/0012148;

R. Rattazzi and A. Zaffaroni, Comments on the holographic picture of the Randall-Sundrum model, JHEP 04 (2001) 021 hep-th/0012248]; 
M. Pérez-Victoria, Randall-Sundrum models and the regularized AdS/CFT correspondence, JHEP 05 (2001) 064 hep-th/0105048;

T. Gherghetta, Warped models and holography, hep-ph/0601213.

[2] L. Randall and R. Sundrum, A large mass hierarchy from a small extra dimension, Phys. Rev. Lett. 83 (1999) 3370 hep-ph/9905221;

A. Pomarol, Gauge bosons in a five-dimensional theory with localized gravity, Phys. Lett. B 486 (2000) 153 hep-ph/9911294.

[3] J. Erlich, E. Katz, D.T. Son and M.A. Stephanov, QCD and a holographic model of hadrons, Phys. Rev. Lett. 95 (2005) 261602 hep-ph/0501128];

L. Da Rold and A. Pomarol, Chiral symmetry breaking from five dimensional spaces, Nucl. Phys. B 721 (2005) 79 hep-ph/0501218.

[4] R. Contino, Y. Nomura and A. Pomarol, Higgs as a holographic pseudo-goldstone boson, Nucl. Phys. B 671 (2003) 148 hep-ph/0306259;

R. Barbieri, A. Pomarol and R. Rattazzi, Weakly coupled higgsless theories and precision electroweak tests, Phys. Lett. B 591 (2004) 141 hep-ph/0310285;

R.S. Chivukula, E.H. Simmons, H.-J. He, M. Kurachi and M. Tanabashi, The structure of corrections to electroweak interactions in Higgsless models, Phys. Rev. D 70 (2004) 075008 hep-ph/0406077;

J. Hirn and V. Sanz, The fifth dimension as an analogue computer for strong interactions at the LHC, JHEP 03 (2007) 100 hep-ph/0612239;

G. Panico and A. Wulzer, Effective action and holography in $5 D$ gauge theories, JHEP 05 (2007) 060 hep-th/0703287;

K. Agashe, C. Csáki, C. Grojean and M. Reece, The S-parameter in holographic technicolor models, arXiv:0704.1821.

[5] K. Agashe, R. Contino and A. Pomarol, The minimal composite Higgs model, Nucl. Phys. B 719 (2005) 165 hep-ph/0412089.

[6] M.S. Chanowitz and M.K. Gaillard, The Tev physics of strongly interacting W's and Z's, Nucl. Phys. B 261 (1985) 379.

[7] G.F. Giudice, C. Grojean, A. Pomarol and R. Rattazzi, The strongly-interacting light Higgs, JHEP 06 (2007) 045 hep-ph/0703164.

[8] R. Sekhar Chivukula, N.D. Christensen, B. Coleppa and E.H. Simmons, Unitarity and bounds on the scale of fermion mass generation, Phys. Rev. D 75 (2007) 073018 hep-ph/0702281.

[9] K. Agashe, A. Delgado, M.J. May and R. Sundrum, RS1, custodial isospin and precision tests, JHEP 08 (2003) 050 hep-ph/0308036.

[10] J.M. Cornwall, D.N. Levin and G. Tiktopoulos, Derivation of gauge invariance from high-energy unitarity bounds on the S matrix, Phys. Rev. D 10 (1974) 1145] [Erratum ibid. 11 (1975) 972];

B.W. Lee, C. Quigg and H.B. Thacker, Weak interactions at very high-energies: the role of the Higgs boson mass, Phys. Rev. D 16 (1977) 1519.

[11] C. Csáki, C. Grojean, L. Pilo and J. Terning, Towards a realistic model of higgsless electroweak symmetry breaking, Phys. Rev. Lett. 92 (2004) 101802 hep-ph/0308038].

[12] A. Delgado and A. Falkowski, Electroweak observables in a general 5D background, JHEP 05 (2007) 097 hep-ph/0702234. 
[13] A. Falkowski, About the holographic pseudo-Goldstone boson, Phys. Rev. D 75 (2007) 025017 hep-ph/0610336.

[14] J. Hirn and V. Sanz, (Not) Summing over Kaluza-Kleins, Phys. Rev. D 76 (2007) 044022 hep-ph/0702005.

[15] Y. Hosotani and Y. Sakamura, Anomalous Higgs couplings in the $\mathrm{SO}(5) \times \mathrm{U}(1)_{B-L}$ gauge-Higgs unification in warped spacetime, hep-ph/0703212.

[16] M.S. Chanowitz, M. Golden and H. Georgi, Low-energy theorems for strongly interacting W's and Z's, Phys. Rev. D 36 (1987) 1490.

[17] M.S. Chanowitz and W. Kilgore, Complementarity of resonant and nonresonant strong $W W$ scattering at the LHC, Phys. Lett. B 322 (1994) 147 hep-ph/9311336];

J. Bagger et al., The strongly interacting $W W$ system: gold plated modes, Phys. Rev. D 49 (1994) 1246 hep-ph/9306256;

J.M. Butterworth, B.E. Cox and J.R. Forshaw, $W W$ scattering at the LHC, Phys. Rev. D 65 (2002) 096014 hep-ph/0201098.

[18] E. Accomando, A. Ballestrero, A. Belhouari and E. Maina, Isolating vector boson scattering at the LHC: gauge cancellations and the equivalent vector boson approximation vs complete calculations, Phys. Rev. D 74 (2006) 073010 hep-ph/0608019. 